\title{
On the Pointwise Behavior of Semi-Classical Measures
}

\author{
T. Paul ${ }^{1}$, A. Uribe $^{2, \star}$
}

${ }^{1}$ CEREMADE et CNRS, Université Paris-Dauphine, Place de Lattre de Tassigny, F-75775 Paris Cedex 16, France

${ }^{2}$ Mathematics Department, University of Michigan, Ann Arbor, Michigan 48109, USA

Received: 29 August 1994/in revised form: 24 January 1995

Abstract: In this paper we concern ourselves with the small $\hbar$ asymptotics of the inner products of the eigenfunctions of a Schrödinger-type operator with a coherent state. More precisely, let $\psi_{j}^{\hbar}$ and $E_{j}^{\hbar}$ denote the eigenfunctions and eigenvalues of a Schrödinger-type operator $H_{\hbar}$ with discrete spectrum. Let $\psi_{(x, \xi)}$ be a coherent state centered at the point $(x, \xi)$ in phase space. We estimate as $\hbar \rightarrow 0$ the averages of the squares of the inner products $\left(\psi_{(x, \xi)}^{a}, \psi_{j}^{h}\right)$ over an energy interval of size $\hbar$ around a fixed energy, $E$. This follows from asymptotic expansions of the form

$$
\sum_{j} \varphi\left(\frac{E_{j}(\hbar)-E}{\hbar}\right)\left|\left(\psi_{(x, \xi)}^{a}, \psi_{j}^{\hbar}\right)\right|^{2} \sim \sum_{k=0}^{\infty} c_{k}(a) \hbar^{-n+\frac{1}{2}+k}
$$

for certain test functions $\varphi$ and Schwartz amplitudes $a$ of the coherent state. We compute the leading coefficient in the expansion, which depends on whether the classical trajectory through $(x, \xi)$ is periodic or not. In the periodic case the iterates of the trajectory contribute to the leading coefficient. We also discuss the case of the Laplacian on a compact Riemannian manifold.

\section{Contents}

1. Introduction . . . . . . . . . . . . . . . . 230

2. Coherent States and Hermite Distributions $\ldots \ldots \ldots \ldots \ldots \ldots .238$

3. Semi-Classical Propagation of Coherent States . . . . . . . . . 240

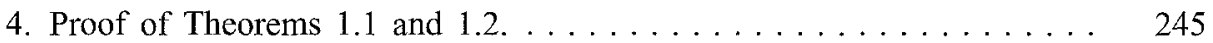

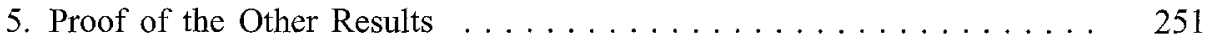

6. The Gaussian Case and Related Poisson Formulas . . . . . . . 252

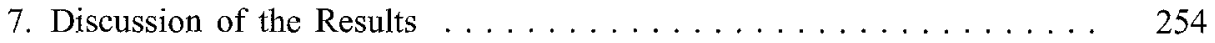

8. References ........................ 257

^ Research supported in part by NSF grant DMS-9303778 


\section{Introduction}

Let $H=-\hbar^{2} \Delta+V(x)$ be a Schrödinger operator with $V$ smooth, on $\mathbb{R}^{n}$ (in which case we assume $V$ tends to infinity at infinity and therefore $H$ has discrete spectrum) or on a compact Riemannian manifold, $M$. The trace formula, [14], describes the small $\hbar$ asymptotics of the average, over a spectral interval of size $\hbar$, of the matrix elements of a semi-classical observable, $b\left(x, \hbar D_{x}\right)$, between eigenvectors of $H$ : Let $\varphi$ be a Schwartz function whose Fourier transform is compactly supported and let $E_{j}^{\hbar}$ and $\psi_{j}^{\hbar}$ the eigenvalues and eigenvectors of $H$. Then, under certain conditions on the Hamilton flow of the Hamiltonian $\mathscr{H}(x, \xi)=\frac{1}{2}|\xi|^{2}+V(x)$ on $\Sigma_{E}=\left\{(\xi, x) ; \frac{1}{2}|\xi|^{2}+V(x)=E\right\}$, we have an asymptotic expansion of the form

$$
\begin{aligned}
& \sum_{j} \varphi\left(\frac{E_{j}(\hbar)-E}{\hbar}\right)\left(\psi_{j}^{\hbar}, b\left(x, \hbar D_{x}\right) \psi_{j}^{\hbar}\right) \\
& \sim\left(\hat{\varphi}(0) \frac{\int_{\Sigma_{E}} b(\xi, x) d \mu^{L}}{\left.(2 \pi)^{-n+1} \hbar^{-(n-1)}+\sum_{k=-n+2}^{+\infty} c_{k}(\hat{\varphi}) \hbar^{k}\right)}\right. \\
& \quad+\sum_{\gamma}\left(\hat{\varphi}\left(T_{\gamma}\right) \frac{e^{i\left(S_{\gamma} / \hbar+\sigma_{\gamma}\right)}}{\sqrt{\left|\operatorname{det}\left(1-P_{\gamma}\right)\right|}} \int_{0}^{T_{\gamma}^{*}} b(x(t), \xi(t)) d t+\sum_{j=1}^{\infty} d_{j}^{\gamma}(\hat{\varphi}) \hbar^{j}\right) .
\end{aligned}
$$

Here:

- $d \mu^{L}$ is the Liouville measure on $\Sigma_{E}$,

- $\gamma$ runs over the periodic trajectories of $\frac{1}{2}|\xi|^{2}+V(x)$ on $\Sigma_{E}$ with periods $T_{\gamma}$ in the support of $\hat{\varphi}$ (the Fourier transform of $\varphi$ ),

- $T_{y}^{*}$ is the primitive period of $\gamma$,

- $S_{\gamma}=\int_{y} \xi d x$

- $\sigma_{y}$ is the Maslov index of $\gamma$,

- $P_{\gamma}$ is the Poincaré mapping of $\gamma$,

- $c_{k}(\cdot)$ are distributions with support in $\{0\}$,

- $d_{j}^{y}(\cdot)$ are distributions with supports in $\left\{T_{\gamma}\right\}$.

Tauberian theorems allow to pass to the limit where $\varphi$ tends to the characteristic function of $[E-c \hbar, E+c \hbar]$. This gives, assuming that the set of periodic points on $\Sigma_{E}$ has measure zero, that

$$
\sum_{\left|E_{j}-E\right| \leqq c \hbar}\left(\psi_{j}^{\hbar}, b\left(x, \hbar D_{x}\right) \psi_{j}^{\hbar}\right)=\frac{2 c}{(2 \pi)^{n}} \int_{\Sigma_{E}} b(x, \xi) d \mu^{L} \hbar^{-(n-1)}+o\left(\hbar^{-(n-1)}\right) .
$$

From this one gets the following result on ergodicity of eigenfunctions: If the flow of $\mathscr{H}$ on $\Sigma_{E}$ is ergodic, then, except for a subsequence of density 0 ,

$$
\lim _{E_{j}^{\hbar} \rightarrow E, \hbar \rightarrow 0}\left(\psi_{j}^{\hbar}, b\left(x, \hbar D_{x}\right) \psi_{j}^{\hbar}\right)=\frac{\int_{\Sigma_{E}} b(x, \xi) d \mu^{L}}{\int_{\Sigma_{E}} d \mu^{L}} .
$$

(For a precise statement see [11].) Another way of writing (3) in $\mathbb{R}^{n}$ is by using the so-called anti-Wick or Toeplitz quantization. Let $\left(\psi_{(x, \xi)}\right),(x, \xi) \in T^{*} \mathbb{R}^{n}$, be the 
family of coherent states:

$$
\psi_{(x, \xi)}(y)=2^{-n / 4}(2 \pi \hbar)^{-\frac{3 n}{4}} e^{-i \frac{\xi x}{2 \hbar}} e^{i \frac{\xi y}{\hbar}} e^{-\frac{(y-x)^{2}}{2 \hbar}}
$$

The anti-Wick quantization of $b(x, \xi), b^{A W}\left(x, \hbar D_{x}\right)$, is the operator defined by the formula

$$
b^{A W}\left(x, \hbar D_{x}\right)=\int b(x, \xi)\left(\psi_{(x, \xi)}, \cdot\right) \psi_{(x, \xi)} d x d \xi .
$$

It is easy to check that, under very general assumptions on $b$ and $V$,

$$
\left(\psi_{j}^{\hbar}, b^{A W}\left(x, \hbar D_{x}\right) \psi_{j}^{\hbar}\right)=\left(\psi_{j}^{\hbar}, b\left(x, \hbar D_{x}\right) \psi_{j}^{\hbar}\right)+O(\hbar)
$$

So (3) can be written as

$$
\lim _{E_{j}^{\hbar} \rightarrow E, \hbar \rightarrow 0} \int b(x, \xi)\left|\left(\psi_{(x, \xi)}, \psi_{j}^{\hbar}\right)\right|^{2} d x d \xi=\frac{\int_{\Sigma_{E}} b(x, \xi) d \mu^{L}}{\int_{\Sigma_{E}} d \mu^{L}} .
$$

In other words, ergodicity of the classical flow on $\Sigma_{E}$ implies that the measures $\left|\left(\psi_{(x, \xi)}, \psi_{j}^{\hbar}\right)\right|^{2} d x d \xi$ converge weakly to the normalized Liouville measure of $\Sigma_{E}$.

Numerical computations, for the so-called billiard problem on the stadium [12], and for the hydrogen atom in a strong magnetic field, [6], show however that some concentration of eigenfunctions near unstable periodic orbits may occur. This scar phenomenon seems to disappear in the classical limit, contrary to the case of modes and quasi-modes associated to stable periodic orbits. of

Our purpose in this paper is to show that, on the average, the pointwise limit

$$
\left|\left(\psi_{(x, \xi)}, \psi_{j}^{\hbar}\right)\right|^{2}
$$

depends strongly on whether or not $(x, \xi)$ belongs to a periodic trajectory, and to analyze its behavior in each case.

Before we state the results precisely, we would like to present the main ideas. The contribution of the periodic trajectories in the trace formula disappears in formula (2), since it appears in (1) at a lower order in $\hbar$. On the other hand, the coefficient of the contribution of $\gamma$ depends strongly on the support of $b(x, \xi)$, so it is natural to think that if one takes symbols whose supports concentrate near a part of a periodic trajectory as $\hbar$ goes to zero, the periodic orbit can make a contribution to the leading order term. Using such symbols amounts, in effect, to observing the wave functions at a smaller scale in phase space. Although this type of symbols does not belong to classical pseudo-differential classes, the anti-Wick quantization allows to consider such singular symbols. The simplest example is a symbol of the form

$$
b_{\left(x_{0}, \check{\xi}_{0}\right)}(x, \xi)=\delta\left(x-x_{0}, \xi-\xi_{0}\right),
$$

a Dirac mass at $\left(x_{0}, \xi_{0}\right)$. Then $(5)$ becomes

$$
b_{\left(x_{0}, \xi_{0}\right)}^{A W}\left(x, \hbar D_{x}\right)=\left(\psi_{\left(x_{0}, \xi_{0}\right)} \cdot \cdot\right) \psi_{\left(x_{0}, \xi_{0}\right)} .
$$


Such an operator is related to the theory of Hermite Fourier integral operators. Formally, it can be viewed as a pseudo-differential operator with Weyl symbol,

$$
B_{\left(x_{0}, \xi_{0}\right)}(x, \xi)=(4 \pi \hbar)^{-n} e^{-\frac{\left(x-x_{0}\right)^{2}+\left(\xi-\xi_{0}\right)^{2}}{\hbar}}
$$

which obviously is not in any standard symbol class. If (3) were still true for $b^{A W}$ given by (9), we would get that for almost all eigenfunctions, the limit as $E_{j} \rightarrow E$ and $\hbar \rightarrow 0$ of $\left|\left(\psi_{(x, \xi)}, \psi_{j}^{\hbar}\right)\right|^{2}$ would be the result of applying the normalized Liouville measure to $B_{\left(x_{0}, \xi_{0}\right)}$, which is

$$
\int B_{\left(x_{0}, \xi_{0}\right)} d \mu^{L}=\frac{(4 \pi \hbar)^{-\frac{1}{2}}}{\left|\nabla \mathscr{H}\left(x_{0}, \xi_{0}\right)\right|}+O(1)
$$

Our main result shows that, on average, there are extra contributions to $\mid\left(\psi_{(x, \xi)}\right.$, $\left.\psi_{j}^{\hbar}\right)\left.\right|^{2}$.

It is useful to express our main result for a more general class of coherent states (see the next section for details).

Preliminary Definition. Let $a \in S\left(R^{n}\right)$, and $(x, \xi) \in R^{2 n}$ or $T^{*} M$. A generalized coherent state centered at $(x, \xi)$ and symbol $a$ is defined locally around $x$ as:

$$
\psi_{(x, \xi)}^{a}(y)=\rho(y-x)(2 \pi \hbar)^{-\frac{3 n}{4}} 2^{-n / 4} e^{-i x \zeta / 2 \hbar} e^{i \xi y / \hbar} \hat{a}\left(\frac{y-x}{\sqrt{\hbar}}\right) .
$$

Here $\rho$ is a $C_{0}^{\infty}$ cutoff function equal to 1 near 0 , and in the manifold case the formula above is in a given coordinate system.

\section{Remarks.}

- The formal definition agrees with this one to leading order in $\hbar$, but it allows for higher order terms which are needed to make the definition coordinateindependent. The Schwartz function $a$ is invariantly a symplectic spinor, which is the symbol of the generalized coherent state, see Sect. 2.

- Proposition 2.4 below shows that the cutoff $\rho$ is semiclassically inessential: modulo $O\left(\hbar^{\infty}\right)$ the state above is independent of it.

- In the case where $a(\eta)=(4 \pi)^{-n / 4} e^{-\left(\eta^{2} / 2\right)}$, this is the usual coherent states definition (up to the inessential cut-off and normalization).

- The normalization in (12) is such that the $L^{2}$ norm of $\psi_{(x, \xi)}^{a}$ is $O\left(\hbar^{-n / 2}\right)$. It is chosen so that the Wigner function of the coherent state (see below) converges, as $\hbar \rightarrow 0$, to a Dirac mass at $(x, \xi)$.

Statement of the Main Results. Let $H_{\hbar}=\sum_{l=0}^{L} \hbar^{l} P_{l}\left(x, D_{x}\right)$, where $P_{l}$ is a differential operator of order $l$ on $\mathbb{R}^{n}$ (or $M$ ) of principal symbol $P_{l}^{0}$, sub-principal symbol $P_{l}^{-1}$ (defined on manifolds if $P_{l}$ is regarded as acting on half-densities) and smooth coefficients. Let $\mathscr{H}(x, \xi)=\sum_{l=0}^{L} P_{l}^{0}(x, \xi)$ and $\mathscr{H}_{s u b}(x, \xi)=\sum_{l=0}^{L} P_{l}^{-1}(x, \xi)$ be the principal and sub-principal symbols of $H_{n}$. We assume that $P_{L}$ is elliptic, $\mathscr{H}$ is positive, and in case $M=\mathbb{R}^{n}$, that $\mathscr{H}$ tends to infinity at infinity. Let $E_{j}^{\hbar}$ and $\psi_{j}^{\hbar}$ denote the eigenvalues and eigenvectors of $H_{\hbar}$. Throughout we will use the 
following normalization of the Fourier transform:

$$
\hat{f}(\xi)=(2 \pi)^{-\frac{n}{2}} \int e^{i \check{\zeta} x} f(x) d x
$$

Theorem 1.1. Let $(x, \xi)$ be a point in $T^{*}\left(\mathbb{R}^{n}\right)$ (or $\left.T^{*}(M)\right)$ not in classical equilibrium, and let $E=\mathscr{H}(x, \xi)$. Assume that $(x, \xi)$ is not periodic with respect to the Hamilton flow of $\mathscr{H}$. Let $\varphi \in C^{\infty}(\mathbb{R})$ with compactly supported Fourier transform, and let $\psi_{(x, \xi)}^{a}$ be defined by (12). Then, as $\hbar \rightarrow 0$,

$$
\sum_{j} \varphi\left(\frac{E_{j}(\hbar)-E}{\hbar}\right)\left|\left(\psi_{(x, \xi)}^{a}, \psi_{j}^{\hbar}\right)\right|^{2} \sim \sum_{k=0}^{\infty} c_{k}(a) \hbar^{-n+\frac{1}{2}+k},
$$

with

$$
c_{0}(a)=\frac{2^{-n / 2}}{\sqrt{2 \pi}}(2 \pi)^{-3 n / 2} \hat{\varphi}(0) \int e^{-i t^{2} \dot{x} \dot{\xi} / 2} e^{i t \eta \dot{x}} \overline{a(\eta)} a(\eta-t \dot{\xi}) d \eta d t .
$$

Moreover, $\forall c>0$,

$$
\begin{aligned}
\sum_{\left|E_{j}(\hbar)-E\right| \leqq c \hbar}\left|\left(\psi_{(x, \xi)}, \psi_{j}^{\hbar}\right)\right|^{2}= & \frac{c}{\pi} 2^{-n / 2}(2 \pi)^{-3 n / 2} \hbar^{-n+\frac{1}{2}} \\
& \times \int e^{-i t^{2} \dot{x} \dot{\xi} / 2} e^{i t \eta \dot{x}} \overline{a(\eta)} a(\eta-t \dot{\xi}) d \eta d t+o\left(\hbar^{-n+\frac{1}{2}}\right) .
\end{aligned}
$$

We will next state the result in the periodic case, in coordinates. If $(x, \xi)$ belongs to a periodic trajectory $\gamma$ of action $S_{\gamma}$ and primitive period $T_{\gamma}>0$, let $S(t)$ be the matrix solution of

$$
\dot{S}(t)=J \operatorname{Hess}(\mathscr{H})(x(t), \xi(t)) \cdot S(t), \quad S(0)=\text { Identity },
$$

where $J$ is the matrix $\left(\begin{array}{cc}0 & -I d \\ I d & 0\end{array}\right),(x(t), \xi(t))$ is the trajectory of the Hamilton flow generated by $\mathscr{H}$ starting at $(x, \xi)$ and Hess( $\mathscr{H}$ ) is the Hessian of $\mathscr{H}$ (see Sect. 3). Invariantly, the mapping defined by $S(t)$ is the differential of the Hamilton flow of $\mathscr{H}$, and $S(t)$ determines, by continuity in $t$, an element of $M p\left(R^{n}\right)$ (starting with the identity element at $t=0$ ). Therefore one can associate to it a unitary operator, $M(S(t))$, on $L^{2}\left(\mathbb{R}^{n}\right)$ through the metaplectic representation. A key role will be played by the metaplectic quantization of $S\left(T_{\gamma}\right)$,

$$
U:=M\left(S\left(T_{\gamma}\right)\right) \text {. }
$$

We will denote

$$
\mathrm{Sub}_{\gamma}:=\int_{0}^{T_{\gamma}} \mathscr{H}_{\text {sub }}(x(t), \xi(t)) d t .
$$

Theorem 1.2. With the above notations, if $(x, \xi)$ belongs to the periodic trajectory $\gamma$,

$$
\sum_{j} \varphi\left(\frac{E_{j}(\hbar)-E}{\hbar}\right)\left|\left(\psi_{(x, \xi)}^{a}, \psi_{j}^{\hbar}\right)\right|^{2} \sim \sum_{k} d_{k}(a) \hbar^{-n+\frac{1}{2}+k}
$$


with

$$
d_{0}=\frac{2^{-n / 2}}{\sqrt{2 \pi}}(2 \pi)^{-3 n / 2} \sum_{l \in \mathbb{Z}} \hat{\varphi}\left(l T_{\gamma}\right) e^{i l\left(\frac{S_{y}}{\hbar}+\mathrm{Sub}_{\gamma}\right)} \int e^{-i t^{2} \dot{x} \dot{\xi} / 2} e^{i t \eta \dot{x}} \overline{a(\eta)}\left(U^{l} a\right)(\eta-t \dot{\xi}) d \eta d t
$$

(the term $l=0$ is precisely the previous coefficient, $c_{0}$ ). Moreover if $\gamma$ has an infinitesimal Poincaré section invariant by the linearized flow, and if the Poincaré mapping of $\gamma$ is diagonalizable over $\mathbb{C}$ and it has at least one hyperbolic summand, then as $\hbar \rightarrow 0$ along the Bohr-Sommerfeld values,

$$
\hbar=\frac{S_{y}}{2 \pi m}, \quad m \rightarrow \infty \text { along integer values }
$$

one has:

$$
\begin{aligned}
\sum_{\left|E_{j}(\hbar)-E\right| \leqq c \hbar}\left|\left(\psi_{(x, \xi)}, \psi_{j}^{\hbar}\right)\right|^{2}= & \hbar^{-n+\frac{1}{2}} \frac{c}{\pi} 2^{-n / 2}(2 \pi)^{-3 n / 2} \\
& \times \int e^{-i t^{2} \dot{x} \dot{\xi} / 2} e^{i t \eta \dot{x}} \overline{a(\eta)} a(\eta-t \dot{\xi}) d \eta d t \\
& +\hbar^{-n+\frac{1}{2}} \frac{1}{\pi} 2^{-n / 2}(2 \pi)^{-3 n / 2} \sum_{l \neq 0} \frac{\sin \left(c l T_{\gamma}\right)}{\left(l T_{\gamma}\right)} e^{i l \mathrm{Sub}_{\gamma}} \\
& \times \int e^{-i t^{2} \dot{x} \dot{\xi} / 2} e^{i t \eta \dot{x}} \overline{a(\eta)}\left(U^{l} a\right)(\eta-t \dot{\xi}) d \eta d t+o\left(\hbar^{-n+\frac{1}{2}}\right),
\end{aligned}
$$

where the series above converges absolutely.

Remark. The result is still valid for any sequence of values of $\hbar$ of the form

$$
\hbar=\frac{S_{\gamma}}{2 \pi(m+\alpha)}, \quad 0 \leqq \alpha \leqq 1,
$$

the formula above being the same after adding $\alpha$ to $\operatorname{Sub}_{\gamma}$. Although we won't prove it here, in fact we believe that the case of $\hbar \rightarrow 0$ continuously follows from simple modifications of our argument.

We will now give an interpretation of the coefficient $c_{0}$. Let

$$
\Omega_{\hbar}:=\left\{j ; E_{j} \in[E-c \hbar, E+c \hbar]\right\} .
$$

Assuming $E$ is a regular value of $\mathscr{H}$ and that almost all points on the energy shell $\mathscr{H}^{-1}(E):=\Sigma_{E}$ are not periodic, the differentiated Weyl law of [14] says that the cardinality of this set is

$$
\# \Omega_{\hbar}=\hbar^{-(n-1)} \frac{\int_{\Sigma_{E}} d \mu^{L}}{(2 \pi)^{n}}+o\left(\hbar^{-(n-1)}\right) .
$$

Proposition 1.3. Assume that almost all points on the energy shell $\Sigma_{E}$ are not periodic. Let $W_{(x, \xi)}^{a}$ be the Weyl symbol of the operator $u \mapsto\left(u, \psi_{(x, \xi)}^{a}\right) \psi_{(x, \xi)}^{a}$ (on a manifold this is in a given coordinate system), that is the Wigner function of 
$\psi_{(x, \zeta)}^{a}(y)$. Then,

$$
\int_{\Sigma_{E}} W_{(x, \xi)}^{a} d \mu^{L}=\frac{\hbar^{-\frac{1}{2}}}{2 \pi}(4 \pi)^{-n / 2} \int e^{-i t^{2} \dot{x} \dot{\xi} / 2} e^{i t \eta \dot{x}} \overline{a(\eta)} a(\eta-t \dot{\xi}) d \eta d t+O\left(\hbar^{-1 / 4}\right),
$$

therefore the first term on the right-hand side of (13) is

$$
\left(\int \varphi\right) \hbar(2 \pi \hbar)^{-n} \int_{\Sigma_{E}} W_{(x, \xi)}^{a} d \mu^{L}+O\left(\hbar^{-n+3 / 4}\right)
$$

In particular:

- If $(x, \xi)$ is not periodic,

$$
\sum_{j \in \Omega_{\hbar}}\left|\left(\psi_{(x, \xi)}, \psi_{j}^{\hbar}\right)\right|^{2}=\neq \Omega_{\hbar} \times\left(\frac{\int W_{(x, \xi)}^{a} d \mu^{L}}{\int d \mu^{L}}+o\left(\hbar^{-\frac{1}{2}}\right)\right) .
$$

- If $(x, \xi) \in \gamma$ and $\gamma$ satisfies the assumptions of Theorem $1.2, \exists v \in \mathbb{R}$ generically non-zero such that

$$
\sum_{j \in \Omega_{\hbar}}\left|\left(\psi_{(x, \xi)}, \psi_{j}^{\hbar}\right)\right|^{2}=\# \Omega_{\hbar} \times\left(\frac{\int W_{(x, \xi)}^{a} d \mu^{L}}{\int d \mu^{L}}+v \hbar^{-\frac{1}{2}}+o\left(\hbar^{-\frac{1}{2}}\right)\right)
$$

for $\hbar$ of the form $\hbar=\frac{S_{y}}{2 \pi m}$, as before.

The next result is an immediate consequence of the preceding and shows a little more precisely the role of periodic trajectories on the pointwise behavior of semiclassical measures.

Corollary 1.4. - Assume $(x, \xi)$ is not periodic. Then $\forall \varepsilon>0$ there exists a subsequence $\left\{E_{j_{k}}\right\} \subset \Omega_{\hbar}$ of positive density such that, for $\hbar$ small enough,

$$
\left|\left(\psi_{(x, \xi)}, \psi_{j}^{\hbar}\right)\right|^{2} \leqq \hbar^{-\frac{1}{2}}\left(\frac{\int W_{(x, \xi)}^{a} d \mu^{L}}{\hbar^{-\frac{1}{2}} \int d \mu^{L}}+\varepsilon\right)
$$

- Assume $(x, \xi) \in \gamma$ with $\gamma$ an unstable trajectory, and suppose moreover that $\frac{2^{-n / 2}}{\pi}(2 \pi)^{-3 n / 2} \sum_{l \neq 0} \frac{\sin \left(c l T_{\gamma}\right)}{\left(l T_{\gamma}\right)} e^{i l S u b_{\gamma}} \int e^{-i t^{2} \dot{\dot{x}} \dot{\xi} / 2} e^{i t \eta \dot{x}} a(\eta)\left(U^{l} a\right)(\eta-t \dot{\xi}) d \eta d t=: b>0$

(this is true in some of the Gaussian examples of Sect. 6). Then $\forall \varepsilon>0$ there exists a subsequence $\left\{E_{j_{k}}\right\} \subset \Omega_{\hbar}$ of positive density such that, for $\hbar$ small enough and of Bohr-Sommerfeld type,

$$
\left|\left(\psi_{(x, \xi)}, \psi_{j}^{\hbar}\right)\right|^{2} \geqq \hbar^{-\frac{1}{2}}\left(\frac{\int W_{(x, \xi)}^{a} d \mu^{L}}{\hbar^{-\frac{1}{2}} \int d \mu^{L}}+\frac{b}{2 c \int d \mu^{L}}-\varepsilon\right) .
$$

We will now give a coordinate-free interpretation of the integral appearing in the $l^{\text {th }}$ term in $(18), \forall l \in \mathbb{Z}$. 
Consider the symplectic vector space $V=T_{(x, \xi)}\left(T^{*} M\right)$; let $M p(V)$ denote the metaplectic group of $V$, double cover of the group of linear symplectic transformations of $V$. Choosing a coordinate system near $x$, we get naturally-induced coordinates of $T^{*} M$ and therefore linear coordinates $(\delta x, \eta)$ on $V$. Given a coherent state $\psi_{(x, \xi)}^{a}$ we claim that, intrinsically, the function $a(\eta)$ should be thought of as a smooth vector in the metaplectic representation of $M p(V)$ (see Sect. 2). Let $\rho$ be the Weyl representation of the Heisenberg group of $V$. Explicitly, acting on functions of $\eta, d \rho$ is the representation

$$
\left\{\begin{array}{l}
d \rho\left(\delta x_{j}\right)(a)(\eta)=\eta_{j} a(\eta) \\
d \rho\left(\eta_{j}\right)(a)(\eta)=-i \frac{\partial}{\partial_{\eta_{j}}} a(\eta)
\end{array}\right.
$$

Then the integral

$$
\int e^{-i t^{2} \dot{x} \dot{\xi} / 2} e^{i t \eta \dot{x}}\left(U^{l} a\right)(\eta-t \dot{\xi}) d t
$$

is the projection of $U^{l} a$ onto the space of tempered distributions $f$ satisfying

$$
d \rho(\Xi)(f)=0,
$$

where

$$
\Xi=(\dot{x}, \dot{\xi})
$$

is the vector tangent to the trajectory at $(x, \xi)$. Indeed the operator

$$
\mathscr{G}: \mathscr{P}\left(\mathbb{R}^{n}\right) \ni b \mapsto\left(\eta \mapsto \int_{-\infty}^{\infty} e^{-i t^{2} \dot{x} \dot{\xi} / 2} e^{i t \eta x} b(\eta-t \dot{\xi}) d t\right) \in \mathscr{S}^{\prime}\left(\mathbb{R}^{n}\right)
$$

is precisely $\int \rho(t \exp (\Xi)) d t$ (the integral should be understood in the weak sense). With this notation, the integral appearing in the $l^{\text {th }}$ term in (18) is

$$
\left(\bar{a}, \mathscr{G}\left(U^{l} a\right)\right),
$$

where the outer parentheses denote the pairing between $\mathscr{S}\left(\mathbb{R}^{n}\right)$ and $\mathscr{S}^{\prime}\left(\mathbb{R}^{n}\right)$.

The Riemannian case. We finish this introduction by observing that the previous results apply in particular to the large eigenvalue asymptotics of the eigenfunctions of the Laplacian on a compact Riemannian manifold. Let $M$ be a Riemannian manifold, $\Delta$ the (negative) Laplacian on $M$ and $\lambda_{j}, \psi_{j}$ the eigenvalues and eigenfunctions of $\Delta$. Instead of working with $\hbar^{2} \lambda_{j}$ it is customary in the Riemannian context to work with the square roots of the eigenvalues

$$
\mu_{j}=\sqrt{-\lambda_{j}},
$$

which can be done with trivial modifications to the proof. Pick $(x, \xi) \in S^{*} M$, the unit cotangent bundle of $M$, periodic with respect to geodesic flow. Let $(r, s), r=$ $\left(r_{1}, \ldots, r_{n-1}\right)$ be Fermi coordinates in a neighborhood of $x$, adapted to the geodesic $\gamma$ through $(x, \xi)$. Thus if $(r, s, \varrho, \sigma)$ are the coordinates induced on $T^{*} M$, locally the geodesic $\gamma$ is the parametrized curve $\{r=0, \varrho=0, s=t, \sigma=1\}$. Let $V$ be the tangent space to $T^{*} M$ at $(x, \xi)$. The coordinates $(r, s, \varrho, \sigma)$ induce linear coordinates $\left(\delta r, \delta s, \eta_{r}, \eta_{s}\right)$ on $V$, and in these coordinates the vector $\Xi_{(x, \xi)}$ is $(0, \ldots, 1,0, \ldots, 0)$ ( 1 in the $n^{\text {th }}$ entry). Recall the interpretation given above of the coefficient (18). 
We were led to consider the operator $\mathscr{G}=\int_{-\infty}^{\infty} \rho(\exp (t \Xi)) d t$ mapping Schwartz functions of the variable $\eta=\left(\eta_{r}, \eta_{s}\right)$ to tempered distributional functions of $\eta$, where $\rho$ is the Weyl representation of the Heisenberg group of $V,(25)$. Since $d \rho(\Xi)=i \eta_{s}$ in the present case, the operator $\mathscr{G}$ is basically multiplication by the Dirac delta of $\eta_{s}$,

$$
\mathscr{G}(a)(\eta)=2 \pi \delta\left(\eta_{s}\right) a(\eta) .
$$

Therefore, our main theorem (in the periodic case) takes on the following form:

Theorem 1.5. Assume the geodesic through $(x, \xi)$ is periodic, of primitive length $L$. Let $\chi$ be a smooth cut-off function in the Fermi coordinate chart. Then, for every test function $\varphi$ with Fourier transform in $C_{0}^{\infty}(\mathbb{R})$, and every Schwartz function $a \in \mathscr{S}\left(\mathbb{R}^{n}\right)$,

$$
\begin{aligned}
\sum_{j} \varphi\left(\mu_{j}-\tau\right)\left|\int \overline{\psi_{j}(r, s)} e^{i \tau s} \hat{a}(\sqrt{\tau} r, \sqrt{\tau s}) \chi(r, s) d r d s\right|^{2} \\
=\tau^{\frac{n}{4}-\frac{1}{2}} 2^{1-\frac{n}{4}}(2 \pi)^{-3 n / 4} \sum_{l \in \mathbb{Z}} \hat{\varphi}(l L) e^{-i \tau l L} \int \overline{a\left(\eta_{r}, 0\right)}\left(U^{l} a\right)\left(\eta_{r}, 0\right) d \eta_{r} \\
\quad+O\left(\tau^{\frac{n}{4}-1}\right)
\end{aligned}
$$

as $\tau \rightarrow \infty$ (one has in fact a full asymptotic expansion in powers of $\sqrt{\tau}$ ).

We won't bother to formally state the formula regarding

$$
\sum_{j ;\left|\mu_{j}-\tau\right| \leqq c}\left|\int \overline{\psi_{j}(r, s)} e^{i \tau s} \hat{a}(\sqrt{\tau} r, \sqrt{\tau} s) \chi(r, s) d r d s\right|^{2}
$$

in case $\gamma$ is unstable.

Formula (29) simplifies for certain choices of test functions $a$, as we will now see. Recall that the operator $U$ is the metaplectic quantization of the differential of geodesic flow at $(x, \xi)$, at time $L$. Such a differential leaves invariant both $\Xi$ and the radial direction in $T^{*} M$. Those two directions span a symplectic subspace $V_{1}$ of $V$. Let $V_{2}$ be the symplectic orthogonal to $V_{1}$. Then the differential of the flow preserves this decomposition of $V$; it is the identity on $V_{1}$ and the linearized Poincare map on $V_{2}$. Accordingly, it is natural to consider Schwartz functions $a$ of the form

$$
a(\eta)=e^{-\eta_{s}^{2} / 2} a_{1}\left(\eta_{r}\right)
$$

On such an $a$, the operator $U$ has the form

$$
U a(\eta)=e^{-\eta_{s}^{2} / 2} U_{P}\left(a_{1}\right)\left(\eta_{r}\right),
$$

where $U_{P}$ is the metaplectic quantization of the linearized Poincare map of $\gamma$. On such amplitudes, our formula becomes

$$
\begin{aligned}
& \sum_{j} \varphi\left(\mu_{j}-\tau\right)\left|\int \overline{\psi_{j}(r, s)} e^{\tau\left(i s-s^{2} / 2\right)} \hat{a}_{1}(\sqrt{\tau} r) \chi(r, s) d r d s\right|^{2} \\
& =\tau^{\frac{n}{4}-\frac{1}{2}} 2^{1-\frac{n}{4}}(2 \pi)^{-3 n / 4} \sum_{l \in \mathbb{Z}} \hat{\varphi}(l L) e^{-i \tau I L} \\
& \times \int \overline{a_{1}\left(\eta_{r}\right)}\left(U_{P}^{l} a_{1}\right)\left(\eta_{r}\right) d \eta_{r}+O\left(\tau^{\frac{n}{4}-1}\right) .
\end{aligned}
$$


This can be simplified further. Recall that the Fourier transform $\mathscr{J}$ is the metaplectic quantization of a certain element $J$ of the metaplectic group such that $J^{2}=-I$. Let

$$
\mathscr{M}:=\mathscr{J} U_{P} \mathscr{J}^{-1} \text {. }
$$

This is nothing but the metaplectic quantization of the Poincare map of $\gamma$ on $L^{2}$ functions of the variable $\delta r$. Since $\mathscr{J}$ is unitary, we have:

Corollary 1.6. In the Riemannian context described above, for every Schwartz function $b \in \mathscr{S}\left(\mathbb{R}^{n-1}\right)$ and every test function $\varphi$ with Fourier transform in $C_{0}^{\infty}(\mathbb{R})$, we have

$$
\begin{aligned}
& \sum_{j} \varphi\left(\mu_{j}-\tau\right)\left|\int \overline{\psi_{j}(r, s)} e^{\tau\left(i s-s^{2} / 2\right)} b(\sqrt{\tau} r) \chi(r, s) d r d s\right|^{2} \\
& \quad=\tau^{\frac{n}{4}-\frac{1}{2}} 2^{1-\frac{n}{4}}(2 \pi)^{-3 n / 4} \sum_{l \in \mathbb{Z}} \hat{\varphi}(l L) e^{-i \tau l L} \int \overline{b(r)} \mathscr{M}^{l}(b)(r) d r+O\left(\tau^{\frac{n}{4}-1}\right) .
\end{aligned}
$$

Although we won't go into details here, we mention that the operator $\mathscr{M}$ can be computed in terms of the transverse Jacobi fields of $\gamma$.

The paper is organized as follows: Sects. 2 and 3 deal with propagation of coherent states, Sect. 4 contains the proof of Theorems 1.1 and 1.2 and Sect. 5 the proof of additional results. In Sect. 6 we treat the case of Gaussian symbols and show that the elliptic case gives rise to "Poisson formulae." We conclude in Sect. 7 by a discussion of the results.

\section{Coherent States and Hermite Distributions}

Let $S$ a Riemannian manifold. In [1] (see also [8]) Boutet de Monvel and Guillemin associate to any conic isotropic manifold $\Gamma$ in $T^{*} S$ a family of distributions on $S$ whose wave-front sets are included in $\Gamma$. These distributions have symbols that are symplectic spinors on $\Gamma$. We will concentrate in this paper in the case where $S=M \times \mathbb{R}$, with $M$ an $n$-dimensional Riemannian manifold ( $M$ might be $\mathbb{R}^{n}$ ) and $\Gamma$ is one dimensional. We will work on a local system of coordinates, but, by the theory of Hermite distributions, the main results are independent of any choice of coordinates.

We begin by briefly recalling the definition of Hermite distributions as it applies to the present setting. Let $a(x, \tau, \eta) \in C^{\infty}\left(M \times \mathbb{R}^{+} \times \mathbb{R}^{n}\right)$ compactly supported in $x$ and rapidly decreasing in $\eta$ admitting, as $\tau \rightarrow \infty$, an asymptotic expansion of the following form:

$$
a(x, \tau, \eta) \sim \sum_{j=0}^{\infty} \tau^{-j / 2} a_{j}(x, \eta),
$$

where $\forall j$ the function $a_{j}$ is in the class $C_{0} \mathscr{S}\left(\mathbb{R}^{n} \times \mathbb{R}^{n}\right)$ defined as follows:

Definition 2.1. We'll denote by $C_{0} \mathscr{S}\left(\mathbb{R}^{+} \times \mathbb{R}^{n}\right)$ the set of all smooth functions $a(x, \eta)$ that are compactly supported in $x$ and satisfy: $\forall K, M, N$ non-negative integers $\exists C_{K M N}>0$ such that

$$
\forall(x, \eta) \in \mathbb{R}^{n} \times \mathbb{R}^{n} \quad\left|\eta^{K} \partial_{x}^{M} \partial_{n}^{N} a\right| \leqq C_{K M N} .
$$

(For the precise meaning of (34) see [1] Sect. 3.) 
For $(x, \xi, \alpha) \in T^{*}(M) \times \mathbb{R}$, let $I_{x, \xi, \alpha}^{a}$ be the distribution defined locally by the oscillatory integral

$$
I_{x, \xi, \alpha}^{a}(y, \theta)=\int_{R^{n} \times R^{+}}(2 \pi)^{\frac{3 n}{2}} \tau^{\frac{n}{4}} e^{-i \tau(\theta+\alpha-\xi y)} e^{i \eta(y-x)} a\left(y, \tau, \frac{\eta}{\sqrt{\tau}}\right) d \eta d \tau .
$$

By definition this is a Hermite distribution associated to

$$
\Gamma=\{(\theta, y ; \tau, p) ; \theta=-\alpha+y \cdot \xi, y=x, p=\tau \xi\} .
$$

In particular:

Proposition 2.2. The wave front of $I_{x, \xi, \alpha}^{a}$ is contained in $\Gamma$.

To $a, x, \xi$ we associate as in [14] the following family of functions on $M$ (actually the inverse Fourier transform in $\theta$ of $\left.I_{x, \xi, \alpha=\frac{x_{\xi}^{\xi}}{2}}^{a}\right)$ :

$$
\psi_{(x, \xi)}^{a}(y)=(2 \pi)^{-n} \tau^{\frac{n}{4}} e^{-i \tau\left(\frac{x \bar{\zeta}}{2}-\xi y\right)} \int_{R^{n}} e^{i \eta(y-x)} a\left(y, \tau, \frac{\eta}{\sqrt{\tau}}\right) d \eta .
$$

Definition 2.3. The family $\left\{\psi_{(x, \zeta)}^{a}\right\}_{\tau}$ is called a coherent state or wave packet centered at $(x, \xi)$ and of symbol $a$.

Each $\psi_{(x, \xi)}^{a}$ is a compactly supported $C^{\infty}$ function. As we showed in [14], the previous proposition implies the following:

Proposition 2.4. The frequency set (or micro-support) of $\psi_{(x, \xi)}^{a}$ is $\{(x, \xi)\}$.

Coherent states are localized in space around $x$ to the extent that, to leading order, the $y$ dependence of the amplitude $a$ can be suppressed. This fact will be very useful in what follows, and it reconciles the definition above with the preliminary one introduced in Sect. 1:

Proposition 2.5. Let $a(y, \tau, \eta)$ be a Hermite amplitude satisfying (34). Let $\rho$ be any $C_{0}^{\infty}$ function identically one near the origin in $\mathbb{R}^{n}$. Then

$$
\psi_{(x, \xi)}^{a}(y)=\rho(y-x)(2 \pi)^{-n} \tau^{\frac{n}{4}} e^{-i \tau\left(\frac{x \zeta}{2}-\xi y\right)} \int_{\mathbb{R}^{n}} e^{i(y-x) \eta} a_{0}(x, \eta / \sqrt{\tau}) d \eta+O\left(\tau^{n / 4-1 / 2}\right)
$$

uniformly on compact sets.

Proof. By the estimates (34), it is easy to see that one has (39) with the right-hand side replaced by

$$
\tau^{n / 4} \rho(y-x) e^{-i \tau\left(\frac{x \xi}{2}-\xi y\right)} \int_{\mathbb{R}^{n}} e^{i(y-x) \eta} a_{0}(y, \eta / \sqrt{\tau}) d \eta
$$

To go from here to (39), we do a Taylor expansion of $a$ in $y$ near $y=x$ and we integrate by parts. Specifically, write

$$
a_{0}(y, \eta)=a_{0}(x, \eta)+\sum_{j=1}^{n}\left(y_{j}-x_{j}\right) g_{j}(y, \eta)
$$


where the $g_{j}$ are smooth functions. We must estimate, $\forall j$,

$$
I_{j}(y, \tau)=\int e^{i(y-x) \eta}\left(y_{j}-x_{j}\right) g_{j}(y, \eta / \sqrt{\tau}) d \eta
$$

Evidently

$$
\begin{aligned}
I_{j} & =i \int e^{i(y-x) \eta} \frac{\partial}{\partial \eta_{j}}\left(g_{j}(y, \eta / \sqrt{\tau})\right) d \eta \\
& =\frac{i}{\sqrt{\tau}} \int e^{i(y-x) \eta} \frac{\partial g_{j}}{\partial \eta_{j}}(y, \eta / \sqrt{\tau}) d \eta,
\end{aligned}
$$

which is clearly $O\left(\tau^{(n-1) / 2}\right)$ uniformly on compacts.

Observation. If $b$ is the inverse Fourier transform of the Schwartz function $\eta \mapsto$ $a_{0}(x, \eta)$, then in a neighborhood of $x$ the coherent state above equals

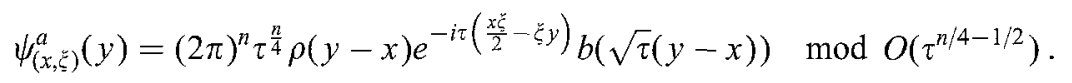

\section{Corollary 2.6.}

$$
\left\|\psi_{(x, \xi)}^{a}\right\|_{L^{2}(M)}^{2}=(2 \pi \hbar)^{-n}\left\|a_{0}(x, \cdot)\right\|_{L^{2}\left(\mathbb{R}^{n}\right)}^{2}+O\left(\hbar^{-n+1}\right) .
$$

Moreover, if $(x, \xi) \neq\left(x^{\prime}, \xi^{\prime}\right)$,

$$
\left(\psi_{(x, \xi)}^{a}, \psi_{\left(x^{\prime}, \xi^{\prime}\right)}^{a^{\prime}}\right)_{L^{2}(M)}=O\left(\hbar^{\infty}\right),
$$

and for any compact $\Omega$ containing $x$

$$
\left(\psi_{(x, \xi)}^{a}, \psi_{(x, \xi)}^{a^{\prime}}\right)_{L^{2}(M)}-\int_{\Omega} \overline{\psi_{(x, \xi)}^{a}(y)} \psi_{(x, \xi)}^{a^{\prime}}(y) d y=O\left(\hbar^{\infty}\right) .
$$

We will omit the straightforward proof.

\section{Semi-Classical Propagation of Coherent States}

Let

$$
\psi_{(x, \xi)}^{a}(y)=(2 \pi)^{-\frac{3 n}{4}}(2 \pi \hbar)^{-\frac{n}{4}} \rho(y-x) e^{-i x \xi / 2 \hbar} \varepsilon^{i \xi y / \hbar} \int a(\sqrt{\hbar} \eta) e^{i \eta(y-x)} d \eta
$$

be a coherent state at $(x, \xi)$. The next theorem shows how such a state evolves under the Schrödinger equation. Let

$$
H_{\hbar}=\sum_{l=0}^{L} \hbar^{l} P_{l}\left(x, D_{x}\right), \quad \mathscr{H}(x, \xi)=\sum_{l=0}^{L} P_{l}^{0}(x, \xi), \quad \mathscr{H}_{\text {sub }}(x, \xi)=\sum_{l=0}^{L} P_{l}^{-1}(x, \xi)
$$

and $S(t)$ be as in Sect. $1 . S(t)$ is the matrix of the differential of the Hamilton flow in coordinates. The associated linear transformation is symplectic and maps the tangent vector to the trajectory at $(x(0), \xi(0))$ to the one at $(x(t), \xi(t))$. Since $S(0)$ is the identity, one can naturally lift the $S(t)$ to the metaplectic group, $M_{P}\left(\mathbb{R}^{n}\right)$ in a continuous way, starting at the identity. We will continue to denote the lift by $S(t)$. Let $M(S(t))$ be the family of unitary operators image of $S(t)$ by the metaplectic representation. 
The following result shows that after evolution a coherent state remains a coherent state and gives the leading term of the symbol.

Theorem 3.1. Under the previous assumptions, $\forall t \in \mathbb{R}$ there exists a symbol a $(t)$ of the form (34) depending smoothly in $t$ and such that

$$
e^{-i \hbar^{-1} t H_{\hbar}}\left(\psi_{(x, \xi)}^{a}\right)=e^{i\left(\ell(t) / \hbar+\int_{0}^{t} \mathscr{H}_{s u b}(x(s), \check{\zeta}(s)) d s\right.} \psi_{(x(t), \bar{\zeta}(t))}^{a(t)} \bmod \left(\hbar^{\infty}\right)
$$

uniformly one each compact in $(t, x)$-space. Here

$$
\mathscr{\ell}(t)=\int_{0}^{t}\left(\frac{\xi \dot{x}-x \dot{\xi}}{2}-\mathscr{H}(x, \xi)\right) d t
$$

Moreover, the leading term of a evolves according to

$$
a_{0}(t)=M(S(t))\left(\left.a_{0}\right|_{t=0}\right) .
$$

The proof of this theorem will be based on the theorem of propagation of Hermite distributions by Fourier integral operators, namely Theorem 7.5 in [1]. We will consider the distribution on $M \times S^{1}$ whose Fourier coefficients are precisely the 1.h.s. of Theorem 3.1, with $\hbar=1 /(m+c)$. This distribution satisfies a certain equation, which we analyze. Then we will show that the solution of this equation is mircolocally equal, in the region of interest, to a Hermite distribution whose Fourier coefficients are given by the right-hand side of the theorem. Finally we will identify the symbol of this Hermite distribution. These ideas have been used in the compact case in [14], but we give an independent proof.

To $H_{\hbar}$ we associate the following family of operators on $M \times S^{1}$ :

$$
A=\sum_{l=0}^{L} D^{-l+L} P_{l}\left(x, D_{x}\right) \text {, }
$$

where $D=D_{\theta}+c, c \in[0,1]$ a parameter. $A$ is a differential operator of order $L$.

We break the proof in a series of lemmas. The first one is straightforward:

Lemma 3.2. Let

$$
\psi_{\tau}^{t}(y)=e^{-i t \tau H_{1 / \tau}}\left(\psi_{(x, \xi)}^{a}\right)(y)
$$

and let

$$
u(t, y, \theta)=\sum_{\tau=1}^{\infty} e^{i \tau \theta} \psi_{\tau+c}^{t}(y)
$$

Then $u(t)$ is a distribution that satisfies

$$
-D^{L-1} D_{t} u=A u
$$

with initial condition

$$
\left.u\right|_{t=0}=I_{x, \xi, \frac{x \zeta}{2}}^{a}
$$

Next, we use $(52,53)$ to control the wave-front set of $u$. 
Lemma 3.3. Let $u$ be a distribution on $\mathbb{R} \times M \times S^{1}$ satisfying (52) and (53). Then its wave-front set is contained in the set

$$
\left\{(t, y, \theta ; \varepsilon, \eta, \tau) ; \tau \neq 0,(y, \eta / \tau)=\phi_{t}(x, \xi / \tau), \theta=\frac{x \xi}{2}+\ell(t), \varepsilon=-\tau \mathscr{H}(x, \xi / \tau)\right\}
$$

Proof. Observing that $u$ satisfies the differential equation $\left(D^{L-1} D_{t}+A\right)(u)=0$, one knows that the wave-front set of $u$ is contained in the characteristic variety of the operator

$$
Q:=D^{L-1} D_{t}+A
$$

which is the set

$$
\operatorname{Char}(Q)=\left\{(t, y, \theta ; \varepsilon, \eta, \tau) ; \tau_{\varepsilon}^{L-1}=-\sum_{l=0}^{L} \tau^{L-1} P_{l}^{0}(y, \eta)\right\} .
$$

Observe that

$$
\operatorname{Char}(Q) \cap\{\tau \neq 0\}=\{(t, y, \theta ; \varepsilon, \eta, \tau) ; \varepsilon / \tau=-\mathscr{H}(y, \eta / \tau)\} .
$$

Since the principal symbol $\sigma_{Q}$ of $Q$ and the function $\tau$ Poisson commute, it follows from (56) that the null-bicharacteristic strips of $Q$ in the region $\{\tau \neq 0\}$ are the same as the trajectories of the Hamilton flow of the function $F=\mathscr{H}(y, \eta / \tau)+\varepsilon / \tau$. We also know that the wave-front set of $u$ is invariant under the Hamilton flow of the principal symbol of $Q$ on $T^{*}\left(M \times S^{1}\right)$. In the region $\{\tau \neq 0\}$ the Hamilton flow of $F$ is, up to a rescaling, the Hamilton flow of $\mathscr{H}$. From this, using the fact that the initial condition has wave-front in the set $\Gamma$ of (37) and the calculus of wave-front sets, one can show that the wave-front set of $u$ is in fact contained in (54).

Lemma 3.4. Let

$$
\widehat{\Sigma_{E}}=\left\{(t, y, \theta ; \varepsilon, \eta, \tau) \in T^{*}\left(M \times S^{1}\right) ; \tau \neq 0, \varepsilon / \tau=-\mathscr{H}(y, \eta / \tau)\right\} .
$$

Then there exists a conic neighborhood of $\widehat{\Sigma_{E}}, \Omega$, contained in $T^{*}(\mathbb{R} \times M \times$ $\left.S^{1}\right) \backslash\{\tau \neq 0\}$, and a classical, first-order pseudodifferential operator on $M \times S^{1}, B$, such that $\hat{Q}:=D^{L-1} D_{t}-D^{L-1} B$ and $Q$ are microlocally equal on $\Omega$. Moreover $\left[B, D_{\theta}\right]=0$.

Proof. It suffices to construct a first order pseudodifferential operator on $M \times S^{1}$ commuting with $D_{\theta}$ and such that $A$ and $D^{L-1} B$ are microlocally equal in a neighborhood of the set

$$
\left\{(y, \theta ; \eta, \tau) \in T^{*}\left(M \times S^{1}\right) ; \tau \neq 0, \mathscr{H}(y, \eta / \tau)=E\right\} .
$$

The following lemma is truly the heart of the proof.

Lemma 3.5. Let $u(t)$ a solution of (52) and (53). Then there exists a Hermite distribution $I_{x(t), \xi(t), \ell(t)}^{a}(t)$ such that:

$$
\left(u(t)-I_{x(t), \xi(t), \ell(t)}^{a}(t)\right) \in C^{\infty} .
$$

Here $(x(t), \xi(t))=\phi_{t}(x, \xi)$ is the trajectory of $(x, \xi)$ under the Hamilton flow of $\mathscr{H}(x, \xi)$ and $\ell(t)$ is as in Theorem 3.1. 
Proof. On one hand, by the previous lemmas,

$$
g:=\hat{Q}(u) \in C^{\infty} .
$$

Therefore, the distribution $u$ can also be described as the solution of the pseudodifferential equation

$$
\begin{aligned}
& \hat{Q}(u)=g, \\
& \left.u\right|_{t=0}=I_{x, \xi, \frac{\xi \xi}{2}}^{a} .
\end{aligned}
$$

On the other hand, one can construct a Fourier integral operator $U(t)$ solving:

$$
\begin{aligned}
D_{t} U(t) & =B U(t) \bmod C^{\infty}, \\
U(0) & =\text { identity },
\end{aligned}
$$

even case $M$ is non-compact, if in the equations above we restrict $|t|$ to be bounded and we restrict $U(t)$ to act on functions supported in a compact set (see for example [17]). In terms of such $U$,

$$
u(t)=\int_{0}^{t} U(t-s)(f) d s+U(t)\left(I_{x, \xi, \frac{\xi \varepsilon}{2}}^{a}\right),
$$

where $f$ is a smooth function satisfying

$$
D^{L-1} f=g .
$$

Such an $f$ indeed exists; observe that therefore the first summand on the right-hand side of (61) is smooth.

To conclude the proof of the lemma we will use the theorem of propagation of Hermite distributions through Fourier integral operators.

$$
I_{x, \xi, \frac{x \xi}{2}}^{a} \text { is a Hermite distribution associated to } \Gamma=\left\{\left(\theta=-\frac{x \xi}{2}, y=x ; \tau, p=\tau \xi\right)\right\} \subset
$$

$\widehat{\Sigma_{E}} \cdot U(t)$ is a Fourier integral operator associated to the flow generated by the principal symbol of $B$, which equals $\mathscr{H}(x, \xi / \tau)$ in a neighborhood of $\Gamma$. An easy calculation of the Hamilton equations (see [14] for details) shows that this flow maps $\Gamma$ into $\Gamma(t)=\left\{\left(\theta=-\frac{x \xi}{2}+\ell(t), y=x(t) ; \tau, p=\tau \xi(t)\right)\right\}$ By the already cited

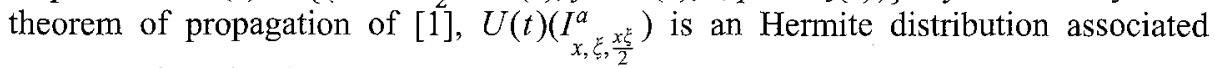
to $\Gamma(t)$. Since by $(61) u(t)$ is equal to it modulo a smooth function, the proof is finished.

To finish the proof of the first part of the theorem just note that by the previous lemma the differences

$$
\psi_{\tau}^{t}-e^{i \tau \ell(t)} \psi_{(x(t), \xi(t))}^{a(t)}
$$

(with $\tau=1,2, \ldots$ ) are the Fourier coefficients in $\theta$ of a smooth function of $M \times S^{1}$; therefore they are rapidly decreasing in $\tau$ uniformly on $x$ in compacts. This proves the first part of the lemma for values of $\hbar$ along the values $\hbar=1 /(m+c), m=$ $1,2, \ldots$ It is clear however that the estimates must be uniform in $c$, and therefore we get the desired conclusion as $\hbar \rightarrow 0$ continuously. 
Finally, we sketch the computation of the principal symbol. A straightforward computation shows that

$$
H_{1 / \tau} \psi_{(x, \xi)}^{a}=\psi_{(x, \xi)}^{a^{\prime}}\left(1+O\left(\tau^{-3 / 2}\right)\right)
$$

with

$$
\begin{aligned}
a^{\prime}= & \mathscr{H}(x, \xi) a+\tau^{-1 / 2}\left(\nabla_{x} \mathscr{H} \cdot D_{\eta}-\nabla_{\zeta} \mathscr{H} \cdot \eta\right) a \\
& +\tau^{-1}\left(\frac{i}{2}\left(D_{\eta}, \eta\right) \text { Hess } \mathscr{H}(x, \xi)\left(D_{\eta}, \eta\right) a+\mathscr{H}_{s u b}(x, \xi) a\right),
\end{aligned}
$$

where

$$
\begin{aligned}
& \left(D_{\eta}, \eta\right) \text { Hess } \mathscr{H}(x, \xi)\left(D_{\eta}, \eta\right) \\
& \quad=\sum_{j, k=0}^{n} \frac{\partial^{2} \mathscr{H}}{\partial x_{j} x_{k}} D_{\eta_{j} \eta_{k}}^{2}+\frac{\partial^{2} \mathscr{H}}{\partial x_{j} \xi_{k}}\left(\eta_{j} D_{\eta_{k}}+\eta_{k} D_{\eta_{j}}\right)+\frac{\partial^{2} \mathscr{H}}{\partial \xi_{j} \xi_{k}} \eta_{j} \eta_{k}
\end{aligned}
$$

is the Weyl quantification of the Hessian of $\mathscr{H}$. Moreover,

$$
\begin{aligned}
& \tau^{-1} D_{t} e^{i\left(\tau \ell(t)+\int_{0}^{t} \mathscr{H}_{s u b}(x(s), \xi(s)) d s\right)} \psi_{(x(t), \xi(t))}^{a(t)} \\
& \quad=e^{i\left(\tau \ell(t)+\int_{0}^{t} \mathscr{H}_{\text {sub }}(x(s), \xi \xi(s)) d s\right)} \psi_{(x(t), \xi(t))}^{a^{\prime}(t)}\left(1+O\left(\tau^{-3 / 2}\right)\right)
\end{aligned}
$$

with

$$
a^{\prime}(t)=\mathscr{H}(x, \xi) a-\tau^{-1 / 2}\left(\dot{\xi} D_{\eta}-\dot{x} \eta\right) a+\tau^{-1}\left(\dot{a}+\mathscr{H}_{\text {sub }}(x, \xi) a\right) .
$$

Identifying term by term gives:

$$
\dot{a}_{0}=\frac{i}{2}\left(D_{\eta}, \eta\right) \text { Hess } \mathscr{H}(x, \xi)\left(D_{\eta}, \eta\right) a_{0} .
$$

We want to prove that a solution of this equation is given by

$$
a_{0}(t)=M(S(t)) a_{0}(0) .
$$

We can easily compute $\frac{d}{d t} M(S(t))$, [13]:

$$
\begin{aligned}
M(S(t+\delta t)) & =M\left(S(t)+\delta t \dot{S}(t)+O\left(\delta t^{2}\right)\right) \\
& =M\left(\left(1+\delta t \dot{S} S^{-1}\right) S\right)+O\left(\delta t^{2}\right) \\
& =M\left(1+\delta t \dot{S} S^{-1}\right) M(S(t))+O\left(\delta t^{2}\right) .
\end{aligned}
$$

Now remark that $1+\delta t \dot{S} S^{-1}$ is the flow of Hamiltonian $(x, \xi)^{t} J \dot{S} S^{-1}(x, \xi)$ modulo $\delta t^{2}$. This implies that

$$
M\left(1+\delta t \dot{S} S^{-1}\right)=e^{i \delta t\left(D_{\eta}, \eta\right) J \dot{S} S^{-1}\left(D_{\eta}, \eta\right)}+O\left(\delta t^{2}\right),
$$

so

$$
\frac{d}{d t} M(S(t))=i\left(D_{\eta}, \eta\right) J \dot{S} S^{-1}\left(D_{\eta}, \eta\right) M(S(t)) .
$$

Identifying once more gives the equation for $S$. 
Remark. It is possible to get the same result directly from the symbolic properties of Hermite distributions. Indeed the family of Hermite distributions constructed before can be considered, taking $t$ as a variable, as a Hermite distribution on $\mathbb{R} \times M \times S^{1}$ associated with the flow-out of $\Gamma$ by the principal symbol of the operator $\left(D_{\theta}^{L-1} D_{t}-\right.$ $A$ ). This new Hermite distribution is in the kernel of the operator $\left(D_{\theta}^{L-1} D_{t}-A\right)$ whose symbol gives rise to a flow tangent to $A$. The transport equation, see [1], gives exactly the result above. We leave this proof to another paper where the case of a general Hermite distribution will be considered.

The Riemannian Case. For the Riemannian case discussed at the end of Sect. 1 one also needs a theorem of propagation of wave packets, which is actually easier to prove; we will be sketchy. Let $M$ be a compact Riemannian manifold, and $\Delta$ the (negative) Laplacian on $M$. The definition of coherent states in the Riemannian case is the same as that of Sect. 2. Consider the operator on $M \times S^{1}$

$$
P:=\sqrt{-\Delta+D^{2}} \text {. }
$$

This is of course a standard first-order $\Psi \mathrm{DO}$, and the required theorem of propagation of wave packets is a consequence of the result that the Fourier integral operator, $\exp ($ it $P)$, maps Hermite distributions of Hermite distributions.

\section{Proof of Theorems 1.1 and $\mathbf{1 . 2}$}

Fix $(x, \xi) \in T^{*} M$, not in classical equilibrium, and $a \in \mathscr{S}\left(\mathbb{R}^{n}\right)$. Denoting $\hbar^{-1}$ by $\tau$, we have:

$$
\begin{aligned}
& \sum_{j} \varphi\left(\frac{E_{j}(\hbar)-E}{\hbar}\right)\left|\left(\psi_{(x, \breve{\zeta})}^{a}, \psi_{j}^{\hbar}\right)\right|^{2}=\frac{1}{\sqrt{2 \pi}} \int \hat{\varphi}(-t)\left(\psi_{(x, \breve{\zeta})}^{a}, e^{-i t \tau(H-E)} \psi_{(x, \xi)}^{a}\right) d t \\
& =\frac{1}{\sqrt{2 \pi}} \int \hat{\varphi}(-t) e^{i\left(\tau \int_{0}^{t}\left(\frac{\xi \dot{x}-x \dot{\xi}}{2}-\mathscr{H}(x, \xi)+E\right) d s+f_{0}^{t} \mathscr{H}_{s u b}(x(s), \bar{\zeta}(s)) d s\right)} \\
& \times\left(\psi_{(x, \xi)}^{a}, \psi_{x(t), \xi(t)}^{a(t)}\right) d t+O\left(\hbar^{\infty}\right) .
\end{aligned}
$$

For simplicity we will take $a(t)=a(t, y, \eta)$ to be the leading term in the expansion (34) of the symbol of Theorem 3.1, and therefore it evolves according to Eq. (48); higher order terms are treated identically.

By Corollary 2.5 we know that $\left(\psi_{(x, \xi)}^{a}, \psi_{(x(t), \xi(t))}^{a(t)}\right)$ is $O\left(\hbar^{\infty}\right)$ if $(x, \xi) \neq(x(t), \xi(t))$, so in (70) it is enough to integrate over intervals around the periods $l T_{\gamma}$ of $\gamma$ if $(x, \xi)$ is periodic and only around zero if it is not. The analysis is therefore localized to a neighborhood of $x$, which enables us to work in a fixed local coordinate system. Let $\beta(t)$ be a cut-off function around zero, and let $f_{l}(t)=\beta\left(t-l T_{\gamma}\right) \hat{\varphi}(-t)$ (only the term $l=0$ arises in the non-periodic case). Since $\mathscr{H}(x, \xi)=E$, we must estimate the integrals

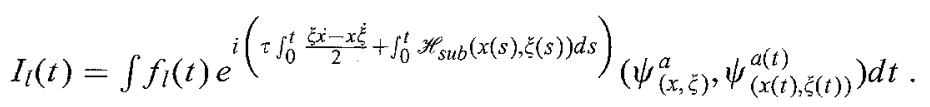


Lemma 4.1. Let

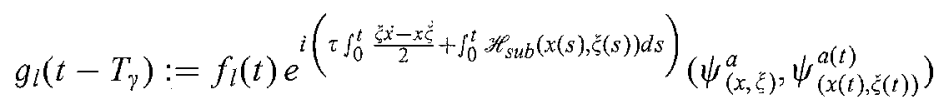

be the integrand in (71). Then, modulo $O\left(\tau^{-\infty}\right), g_{l}(t)$ is of the form

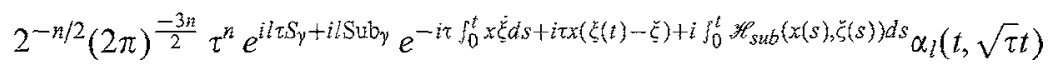

with $\alpha_{1}(t, u) \in C_{0} \mathscr{S}(\mathbb{R} \times \mathbb{R})$ (the class of functions defined in (35)) and such that

$$
\alpha_{l}(0, u)=\hat{\varphi}\left(l T_{\gamma}\right) \int e^{i u y \dot{\xi}(0)} \overline{\hat{a}(y)} \hat{a}^{l T_{\gamma}}(y-u \dot{x}(0)) d y .
$$

Proof. By Proposition 2.4 one can get rid of the cutoffs $\rho$ in $\left(\psi_{(x, \xi)}^{a}, \psi_{(x(t), \xi(t))}^{a^{\prime}}\right)$, since we compute $\alpha \bmod \tau^{-\infty}$. This gives, after some manipulations:

$$
\begin{aligned}
\alpha_{l}(t, \sqrt{\tau} t)= & (2 \pi)^{-n / 2} \beta(t) \hat{\varphi}\left(l T_{\gamma}+t\right) \\
& \times \int e^{i \sqrt{\tau}[y(\xi(t)-\xi)-\eta(x(t)-x)]} e^{i \eta y} \overline{\hat{a}(y)} a^{l T_{\gamma}+t}(\eta) d y d \eta .
\end{aligned}
$$

So

$$
\begin{aligned}
\alpha_{l}(t, u)= & (2 \pi)^{-n / 2} \beta(t) \hat{\varphi}\left(l T_{\gamma}+t\right) \\
& \times \int e^{i t\left[y \frac{\xi(t)-\xi}{t}-\eta \frac{x(t)-x}{t}\right]} e^{i \eta y} \overline{\hat{a}(y)} a^{l T_{\gamma}+t}(\eta) d y d \eta
\end{aligned}
$$

By the stationary phase lemma, since $(\dot{x}, \dot{\xi}) \neq 0$ one can see that $\alpha$ decreases rapidly with $u$. The same argument gives the result for the derivatives of $\alpha$, and the desired uniformity as well.

We have

$$
I_{l} \sim 2^{-n / 2}(2 \pi)^{-\frac{3 n}{2}} \tau^{n} e^{i l\left(\tau S_{y}+S u b_{y}\right)} \int e^{-i \tau \int_{0}^{t} x \dot{\xi} d s+i \tau x(\xi(t)-\xi)+i \int_{0}^{t} \mathscr{H}_{s u b}(x(s), \xi(s)) d s} \alpha_{l}(t, \sqrt{\tau} t) d t .
$$

This integral will be estimated thanks to the following proposition:

Proposition 4.2. Let $\alpha \in C_{0} \mathscr{S}(\mathbb{R} \times \mathbb{R})$ and $\Phi \in C^{\infty}(\mathbb{R})$ satisfying:

$$
\Phi(0)=0=\Phi^{\prime}(0)
$$

Let

$$
I(\tau):=\int e^{i \tau \Phi(t)} \alpha(t, \sqrt{\tau} t) d t
$$

Then:

$$
I(\tau) \sim \tau^{-1 / 2} \sum_{j=0}^{\infty} c_{j} \tau^{-j / 2}
$$

Moreover,

$$
c_{0}=\int e^{i \frac{\phi^{\prime \prime}(0)}{2} t^{2}} \alpha(0, t) d t
$$

Proof. By the assumptions on $\Phi$ there exists a $\Psi \in C_{0}^{\infty}(\mathbb{R})$ such that

$$
\Phi(t)=t^{2} \Psi(t)
$$


in the support of $t \mapsto \alpha(t, u), \forall u \in \mathbb{R}$. Substituting into $I(\tau)$ and making a dilation $s=\sqrt{\tau} t$ we get:

$$
I(\tau)=\tau^{-1 / 2} \int e^{i s^{2} \Phi(s / \sqrt{\tau})} \alpha\left(\frac{s}{\sqrt{\tau}}, s\right) d s .
$$

Since $\alpha$ is rapidly decreasing in the second variable, uniformly with respect to the first, the integrand above is bounded by, say, the integrable function $\left(s^{2}+1\right)^{-1}$ uniformly in $\tau$. Therefore

$$
\lim _{\tau \rightarrow \infty} \tau^{1 / 2} I(\tau)=\int e^{i s^{2} \Psi(0)} \alpha(0, s) d s=c_{0}
$$

To obtain the asymptotic expansion observe that in fact the function of $\sigma$

$$
g(\sigma)=\int e^{i s^{2} \Psi(s \sigma)} \alpha(s \sigma, s) d s
$$

is smooth in a neighborhood of $\sigma=0$ : since $\alpha$ is Schwartz in the second variable uniformly with respect to the first every derivative with respect to $\sigma$ of the integrand in (80) is bounded by an integrable function uniformly in $\sigma$. The expansion (77) is nothing but the Taylor expansion of $g(\sigma)$ around $\sigma=0$.

Remark. If 0 is not a critical point of $\Phi$, then one can easily show that $I(\tau)$ above is $O\left(\tau^{-\infty}\right)$.

We now return to $I_{l}$. Thanks to Lemma $4.1, I_{l}$ can be rewritten as:

$$
\begin{aligned}
I_{l}= & 2^{-n / 2}(2 \pi)^{-\frac{3 n}{2}} \tau^{n} e^{i l\left(\frac{s_{y}}{\hbar}+\operatorname{Sub}_{\gamma}\right)} \\
& \times \int e^{i \tau \Phi(t)} e^{i \int_{0}^{t} \dddot{H} s u b(x(s), \xi(s)) d s} \alpha_{l}(t, \sqrt{\tau} t) d t+O\left(\tau^{-\infty}\right)
\end{aligned}
$$

with

$$
\Phi(t)=-\int_{0}^{t} x(s) \cdot \dot{\xi}(s) d s+x \cdot(\xi(t)-\xi) .
$$

We obviously have $\Phi^{\prime}(t)=(x-x(t)) \cdot \dot{\xi}$. If $(x, \xi)$ is not a periodic point, then as mentioned we only need to consider the term $l=0$, and therefore the asymptotic expansion of Theorem 1, follows from Proposition 4.2.

If $(x, \xi)$ is periodic,

$$
\forall l \in \mathbb{Z} \quad \Phi\left(l T_{\gamma}\right)=0=\Phi^{\prime}\left(l T_{\gamma}\right),
$$

and

$$
\Phi^{\prime \prime}\left(l T_{\gamma}\right)=-\dot{x} \cdot \dot{\xi} .
$$

This means that each integral $I_{I}(\tau)$ has, by Proposition 4.2, an asymptotic expansion of the form:

$$
I_{l} \sim 2^{-n / 2}(2 \pi)^{-\frac{3 n}{2}} e^{i l\left(\frac{s_{y}}{\hbar}+\operatorname{Sub}_{\gamma}\right)} \tau^{n-\frac{1}{2}} \sum_{j=0}^{\infty} c_{j}^{l} \tau^{-j / 2}
$$

with

$$
c_{0}^{l}=\int e^{-i \frac{x^{\frac{i}{\xi}}}{2} t^{2}} \alpha_{l}(0, t) d t .
$$

Plugging (72) in (84) and summing over $l$ gives the existence of (17) and the leading term (18). 
Tauberian Arguments. To prove (15) and (19) we will use the following Tauberian lemma proved in [2] (see also [3]). Consider an expression of the following form:

$$
\Upsilon_{E, \hbar}^{w}(\varphi)=\sum_{j} w_{j}(\hbar) \varphi\left(\frac{E_{j}(\hbar)-E}{\hbar}\right) .
$$

It will be useful to introduce the following

Notation. We will denote by $\mathscr{R}$ the set of all Schwartz functions on the line with compactly supported Fourier transform.

The Tauberian lemma in question is:

Theorem 4.3. (See [2].) Suppose $w_{j}(\hbar), E_{j}(\hbar), E$ and $Y_{\hbar}^{w}$ itself satisfy all of the following:

1. There exists a positive function $\omega(\hbar)$, defined on an interval $\left(0, \hbar_{0}\right)$, and a functional $\mathscr{F}_{0}$ on $\mathscr{R}$, such that for all $\varphi \in \mathscr{R}$,

$$
\Upsilon_{E, \hbar}^{\omega}(\varphi)=\mathscr{F}_{0}(\varphi) \omega(\hbar)+o(\omega(\hbar)), \quad \hbar \rightarrow 0
$$

(both $\mathscr{F}_{0}$ and $\omega$ depending on $E$, in general).

2. If $f \in \mathscr{R}$ is non-negative, identically one near the origin and of mass one, and if one defines $\forall \mu>0$,

$$
f_{\mu}(r)=\mu^{-1} f(r / \mu)
$$

(so that $\left\{f_{\mu}\right\}$ is an approximate identity i.e. each $f_{\mu}$ is positive, of mass one, and $f_{\mu}(r) \rightarrow \delta(r)$ as $\left.\mu \rightarrow 0\right)$, then $\forall c>0$ the limit

$$
\mathscr{L}(c)=\lim _{\mu \rightarrow 0} \mathscr{F}_{0}\left(f_{\mu} * \chi_{[-c, c]}\right)
$$

exists. Here $\chi_{[-c, c]}$ is the characteristic function of the interval $[-c, c]$. Moreover, $\mathscr{L}$ is continuous on an interval of the form $(0, \varepsilon)$.

3. There exists $a k \in Z$ such that $\hbar^{k}=\mathcal{O}(\omega(\hbar)), \hbar \rightarrow 0$.

4. There exists an $\varepsilon>0$ such that for every $\varphi$ there is a constant $C_{\varphi}$ such that for all $E^{\prime} \in[E-\varepsilon, E+\varepsilon]$ :

$$
\left|\Upsilon_{E^{\prime}, \hbar}^{w}(\varphi)\right| \leqq C_{\varphi} \omega(\hbar)
$$

(rough uniformity in $E$ ).

5. The $w_{j}(\hbar)$ are non-negative and bounded: there exists a constant $C \geqq 0$ such that for all $j$ and all $\hbar, 0<\hbar<\hbar_{0}$ :

$$
0 \leqq w_{j}(\hbar) \leqq C .
$$

6. The eigenvalues $E_{j}(\hbar)$ satisfy the following rough estimate: for each $C_{1}$ there exist constants $C_{2}, N_{0}$ such that $\forall k$

$$
\#\left\{j: E_{j}(\hbar) \leqq C_{1}+k \hbar\right\} \leqq C_{2}\left(\hbar^{-1} k\right)^{N_{0}} .
$$

Define the weighted counting function by

$$
N_{E, c}^{w}(\hbar)=\sum_{\left|x_{j}(\hbar)\right| \leqq c} w_{j}(\hbar),
$$


where

$$
x_{j}(\hbar):=\frac{E_{j}(\hbar)-E}{\hbar} .
$$

Then the conclusion is: $\forall c>0$,

$$
N_{E, c}^{w}(\hbar)=\mathscr{L}(c) \omega(\hbar)+o(\omega(\hbar)), \quad \hbar \rightarrow 0 .
$$

In the present context, we wish to take

$$
\omega_{j}=\sqrt{h}\left|\left(\psi_{(x, \xi)}^{a}, \psi_{j}^{\hbar}\right)\right|^{2} .
$$

With the shown normalization, property (88) is ensured. The function $\omega$ is

$$
\omega(\hbar)=\hbar^{-n+1}
$$

so that hypothesis 3 is automatically satisfied, while the functional $\mathscr{F}_{0}$ is given by Theorems 1.1 and 1.2 so that property (86) is true. The rough estimate on the eigenvalues (89) is certainly true in this case, see e.g. [2]. We need to verify the remaining assumptions 2 and 4 of the Tauberian lemma above.

Assumption 2:

- if $(x, \xi)$ is not periodic, then

$$
\mathscr{F}_{0}(\varphi)=\int_{-\infty}^{+\infty} \varphi(t) d t \int e^{-i t^{2} \dot{x} \dot{\xi}} e^{i t \eta \dot{x}} \overline{a(\eta)} a(\eta-t \dot{\xi}) d \eta d t,
$$

that is $\mathscr{F}_{0}$ is proportional to Lebesgue measure on $\mathbb{R}$. Thus assumption 2 is trivially satisfied, with $\mathscr{L}(c)=2 c$.

- if $(x, \xi)$ is periodic, let us only consider values of $\hbar$ of the form $\hbar=\frac{S_{\gamma}}{2 \pi m}$. Then we must prove that the functional

$$
\mathscr{F}_{0}(\varphi)=\sum_{l} \hat{\varphi}\left(l T_{\gamma}\right) \int e^{-i t^{2} \dot{x} \dot{\xi}} e^{i t \eta \dot{x}} \overline{a(\eta)}\left(U^{l} a\right)(\eta-t \dot{\xi}) d \eta d t
$$

has the required property, and the limit function $\mathscr{L}(c)$ is continuous. This is true under a hypothesis of instability; this is an easy consequence of the following:

Lemma 4.4. Let us suppose that the differential $d\left(\phi_{T}\right)_{(x, \xi)}$ of the classical flow at time $T_{\gamma}$ and at $(x, \xi)$ is diagonalizable over $\mathbb{C}$, and has $r$ elliptic directions of angles $\theta_{j}, j=1, \ldots, r$ and $n-1-r$ hyperbolic directions of Liapunov angles $\mu_{k}, k=1, \ldots, n-1-r$, with $r<n-1$. Let $\mu:=\sup _{k} \mu_{k}$. Then

$$
\left|\int e^{-i t^{2} \dot{x} \dot{\xi} / 2} e^{i \hbar \eta \bar{x}} \overline{a(\eta)}\left(U^{l} a\right)(\eta-t \dot{\xi}) d \eta d t\right| \leqq C e^{-|t| \mu}
$$

Before we prove the lemma let us mention that it easily implies the required continuity property of the functional $\mathscr{F}_{0}$.

Proof. Recall the interpretation of the right-hand side of (97) as a matrix coefficient of $U^{l}=M\left(S\left(T_{\gamma}\right)\right)^{l}$ in the metaplectic representation. Let $(e, f) \in \mathbb{R}^{n}$, we define the Weyl operators as:

$$
Z(e, f) a(\eta)=e^{-i \frac{e f}{2}} e^{i e \eta} a(\eta-f)
$$


Then we have to estimate:

$$
\left|\int\left(a, U^{l} Z(t \dot{x}, t \dot{\xi}) a\right) d t\right|
$$

Under the stated assumptions one can find a linear symplectic transformation $R$ such that

$$
R^{-1} S\left(T_{\gamma}\right) R=\left(\begin{array}{cccccc}
1 & 0 & 0 & \alpha & 0 & 0 \\
0 & \operatorname{Diag}\left(\cos \left(\theta_{j}\right)\right) & 0 & 0 & \operatorname{Diag}\left(-\sin \left(\theta_{j}\right)\right) & 0 \\
0 & 0 & \operatorname{Diag}\left(e^{-\mu k}\right) & 0 & 0 & 0 \\
0 & 0 & 0 & 1 & 0 & 0 \\
0 & \operatorname{Diag}\left(\sin \left(\theta_{j}\right)\right) & 0 & 0 & \operatorname{Diag}\left(\cos \left(\theta_{j}\right)\right) & 0 \\
0 & 0 & 0 & 0 & 0 & \operatorname{Diag}\left(e^{\mu k}\right)
\end{array}\right)
$$

with $\alpha \in \mathbb{R}$. It is therefore sufficient to prove the estimate for the metaplectic quantization of the above matrix. More precisely, let $G:=R^{-1} S\left(T_{\gamma}\right) R$ and $b=b\left(\eta_{0}\right.$, $\left.\eta_{1}, \ldots, \eta_{n-1}\right)$, we have:

$$
\left|\left(a, M\left(S\left(l T_{\gamma}\right)\right) Z(t \dot{x}, t \dot{\xi}) a\right)\right|=\left|\left(b, M\left(G^{l}\right) Z((1,0, \ldots, 0)) b\right)\right| .
$$

By (99) one sees that $M\left(G^{l}\right) Z((1,0, \ldots, 0))=U_{\eta_{0}} U_{\eta_{1}} \ldots U_{\eta_{n-1}}$, where the $U_{\eta_{i}}$ are unitary operators acting on the variable $\eta_{i}$. By the Cauchy-Schwartz inequality one easily deduces that

$$
\left|\left(b, M\left(G^{l}\right) Z((1,0, \ldots, 0)) b\right)\right| \leqq C e^{-\frac{l \mu}{2}} \int u\left(\eta_{j}, e^{-l \mu} \eta_{j}\right) d \eta_{j}
$$

where $\mu=\mu_{j}=\sup _{k}\left\{\mu_{k}\right\}$ and

$$
\begin{aligned}
u\left(\eta_{j}, e^{-i \mu} \eta_{j}\right)= & \sqrt{\int|b(\eta)|^{2} d \eta} \\
& \times \sqrt{\int\left|b\left(\eta_{0}, \eta_{1}, \ldots, e^{-l \mu} \eta_{j}, \ldots\right)\right|^{2} d \eta_{0} \ldots d \eta_{j-1} d \eta_{j+1} \ldots d \eta_{n-1}}
\end{aligned}
$$

With the notation

$$
r_{l}:=C e^{-\frac{l \mu}{2}} \int u\left(\eta_{j}, e^{-l \mu} \eta_{j}\right) d \eta_{j}
$$

let us first suppose $l$ positive. Then

$$
\lim _{l \rightarrow+\infty} e^{\frac{l \mu}{2}} r_{l}=C \int u\left(\eta_{j}, 0\right) d \eta_{j}
$$

so $r_{l} \leqq C^{\prime} e^{-\frac{l \mu}{2}}$ for $l$ large enough. If $l$ is negative then note that

$$
r_{l}=C e^{\frac{l \mu}{2}} \int u\left(e^{\frac{l \mu}{2}} \eta_{j}, \eta_{j}\right) d \eta_{j}
$$

and apply the same argument. 
Finally, we need to establish the rough uniformity in $E$. To do that we must ask the patient reader to go over again the proof of the asymptotic expansions of Theorems 1.1 and 1.2. By (70) and Lemma 4.1, instead of the integral of Proposition 4.2 we must estimate integrals of the form

$$
I_{\Delta}(\tau)=\int e^{i \tau \Phi(t)} e^{i \tau t \Delta} \alpha(t, \sqrt{\tau} t) d t,
$$

where $\Delta=E^{\prime}-E$ ranges in a neighborhood of zero and $\Phi$ is the same phase as before. We have to show that

$$
I_{\Delta}(\tau)=O\left(\tau^{-1 / 2}\right)
$$

uniformly in $\Delta$. Proceeding exactly as in the proof of Proposition 4.2 we are led to the expression

$$
I_{\Delta}(\tau)=\tau^{-1 / 2} \int e^{i s^{2} \Psi(s / \sqrt{\tau})} e^{i s \sqrt{\tau} A} \alpha(s / \sqrt{\tau}, s) d s .
$$

The integral is clearly bounded in $\tau$ uniformly in $\Delta$, again by the rapid decrease of $\alpha$ in the second variable. We note that if $\Delta \neq 0$ the integral is $O\left(\tau^{-\infty}\right)$.

\section{Proof of the Other Results}

The Weyl symbol of the operator $\left(\psi_{(x, \xi)}^{a}, \cdot\right) \psi_{(x, \xi)}^{a}$ is:

$$
W_{\psi_{(x, \xi)}^{a}}^{\hbar}(q, p)=\int e^{-i \frac{p y}{\hbar}} \psi_{(x, \xi)}^{a}(x-y / 2) \psi_{(x, \xi)}^{a}(x+y / 2) d y .
$$

Lemma 5.1.

$$
W_{\psi_{(x, \xi)}^{a}}^{\hbar_{0}}(q, p)=(2 \pi \hbar)^{-n}(4 \pi)^{-n / 2} W_{a}^{1}\left(\frac{q-x}{\sqrt{\hbar}}, \frac{p-\xi}{\sqrt{\hbar}}\right) .
$$

The proof is immediate.

Let us define $\mathscr{W}_{a}:=W_{a}^{\Uparrow}$. We want next to compute

$$
\int W_{\psi_{(x, \xi)}^{a}}^{\hbar}(q, p) d \mu^{L}(q, p)
$$

with $d \mu^{L}(q, p)=\frac{d A_{q, p}}{\mid \nabla \mathscr{H} T}$ the Liouville measure ( $d A_{q, p}$ denotes the surface measure on the energy shell $\Sigma_{E}=\mathscr{H}^{-1}(E)$ induced by the Euclidean metric in the $p, q$ coordinates). Let us define the symplectic Fourier transform $\widehat{\mathscr{W}}_{a}$ of $\mathscr{W}_{a}$ through the formula

$$
\mathscr{W}_{a}(q, p)=(2 \pi)^{-n} \int \widehat{\mathscr{W}}_{a}(u, v) e^{-i(u q-v p)} d u d v .
$$

Then, with $\tau=1 / \hbar$.

$$
\begin{aligned}
\int W_{\psi_{(x, \xi)}^{a}}^{\hbar}(q, p) d \mu^{L}(q, p)= & \left(\frac{\tau}{2 \pi}\right)^{n}(2 \pi)^{-n} \\
& \times \int \widehat{\mathscr{W}}_{\alpha}(u, v) e^{-i(u \sqrt{\tau}(q-x)-v \sqrt{\tau}(p-\xi))} \frac{d A_{q, p}}{|\nabla \mathscr{H}|} d u d v .
\end{aligned}
$$


This integral is easily evaluated by the stationary phase formula; the phase is stationary for

$$
q=x, \quad p=\xi, \quad(u, v)=\lambda\left(-\nabla_{q} H, \nabla_{p} H\right)=\lambda(\dot{\xi}, \dot{x})
$$

with $\lambda$ real. This gives:

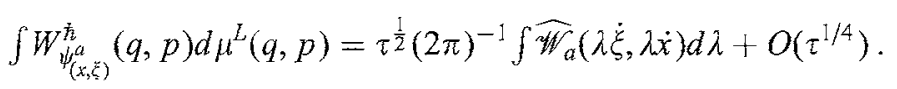

It is well known (see e.g. [7]) that the symplectic Fourier transform of a Wigner function of the type (108) satisfies

$$
\widehat{\mathscr{W}}_{a}(u, v)=(a, Z(u, v) a),
$$

where

$$
Z(v, w) a(y)=e^{-i w v / 2} e^{i v y} a(y-u) .
$$

This gives immediately (as a direct computation also shows) that

$$
\int W_{\psi_{(x, \zeta)}^{a}}^{\hbar}(q, p) d \mu^{L}=\frac{\hbar^{-\frac{1}{2}}}{2 \pi}(4 \pi)^{-n / 2} \int e^{-i t^{2} \frac{x_{\xi}^{\xi}}{2}} e^{i t \eta \dot{x}} a(\eta) a(\eta-t \dot{\xi}) d \eta d t+O\left(\hbar^{-1 / 4}\right) .
$$

The rest of the proposition is clearly obtained from Theorem 1.2.

The proof of Corollary 1.4 is immediate by contradiction. The proof of Theorem 1.5 is obtained by taking $S_{\hbar}=\hbar \sqrt{-\Delta}$; the action of $\gamma$ by homogeneity of the Hamiltonian reduces to the period (if $E=1$ ).

\section{The Gaussian Case and Related Poisson Formulas}

In this section we will show that the leading coefficient of the asymptotic expansion of

$$
\sum_{j} \varphi\left(\frac{E_{j}(\hbar)-E}{\hbar}\right)\left|\left(\psi_{(x, \xi)}^{a}, \psi_{j}^{\hbar}\right)\right|^{2},
$$

given by Theorem 1.3 can be explicitly computed for certain Gaussian symbols $a$.

We first need the lemma:

Lemma 6.1. If $\gamma$ has an infinitesimal Poincaré section invariant by the linearized flow and if the Poincaré mapping of $\gamma$ has $r$ elliptic directions of angles $\theta_{j}$ and $n-1-r$ hyperbolic ones of Liapunov exponent $\mu_{k}$, then one can find a symplectic mapping $R$ such that $R^{-1} S\left(T_{\gamma}\right) R$ is the matrix

$$
\left(\begin{array}{cccccc} 
& 0 & 0 & u & 0 & 0 \\
0 & \operatorname{Diag}\left(\cos \left(\theta_{j}\right)\right) & 0 & 0 & \operatorname{Diag}\left(-\sin \left(\theta_{j}\right)\right) & 0 \\
0 & 0 & \operatorname{Diag}\left(e^{-\mu_{k}}\right) & 0 & 0 & 0 \\
0 & 0 & 0 & 1 & 0 & 0 \\
0 & \operatorname{Diag}\left(\sin \left(\theta_{j}\right)\right) & 0 & 0 & \operatorname{Diag}\left(\cos \left(\theta_{j}\right)\right) & 0 \\
0 & 0 & 0 & 0 & 0 & \operatorname{Diag}\left(e^{\mu_{k}}\right)
\end{array}\right) .
$$


Let us define the Schwartz function $a^{R}:=M(R) a_{0}$, where $a_{0}(\eta)=(4 \pi)^{-n / 4} e^{-\eta^{2} / 2}$. Lemma 6.2. $a^{R}$ is a Gaussian. More precisely, let us write $R=\left(\begin{array}{ll}A & B \\ C & D\end{array}\right)$. Then:

$$
a^{R}(\eta)=\frac{1}{(4 \pi)^{-n / 4} \sqrt{\operatorname{det}(A+i B)}} e^{-\frac{1}{2} \eta^{l}(D-i C)(A+i B)^{-1} \eta} .
$$

Proof. See [13] p. 247, in particular for the definition of the square root.

Proposition 6.3. Under the same hypothesis as in Theorem 1.2,

$$
\sum_{j} \varphi\left(\frac{E_{j}(\hbar)-E}{\hbar}\right)\left|\left(\psi_{(x, \xi)}^{\left.a^{R}\right)}, \psi_{j}^{\hbar}\right)\right|^{2} \sim \sum_{k=0}^{\infty} d_{k}\left(a^{R}\right) \hbar^{-n+\frac{1}{2}+k},
$$

with

$$
d_{0}\left(a^{R}\right)=\frac{(4 \pi)^{-\frac{1}{2}}}{|\nabla \mathscr{H}(x, \xi)|}\left(\sum_{l} e^{i l\left(\frac{S_{y}}{\hbar}+\mathrm{Sub}_{\gamma}+\sigma_{\gamma}-\frac{1}{2} \sum_{j=1}^{r} \theta_{j}\right)} \frac{1}{\sqrt{\prod_{k=1}^{d-1-r} \cosh \left(l \mu_{k}\right)}} \hat{\varphi}\left(l T_{\gamma}\right)\right)
$$

where $\sigma_{\gamma}$ is the Maslov index of $\gamma$. In particular if $(x, \xi)$ is periodic with a Poincaré mapping fully elliptic of angles $\theta_{j}, j=1 \ldots n-1$.

$$
\begin{aligned}
& \sum_{j} \varphi\left(\frac{E_{j}(\hbar)-E}{\hbar}\right)\left|\left(\psi_{(x, \xi)}^{a}, \psi_{j}^{\hbar}\right)\right|^{2} \\
& \quad=\frac{(4 \pi \hbar)^{-\frac{1}{2}}}{T_{\gamma}|\nabla \mathscr{H}(x, \xi)|} \sum_{k} \varphi\left(\frac{1}{T_{\gamma}}\left(k 2 \pi-\frac{S_{\gamma}}{\hbar}+\operatorname{Sub}_{\gamma}+\sigma_{\gamma}+\frac{1}{2} \sum_{j=1}^{n-1} \theta_{j}\right)\right)+O\left(\hbar^{\frac{1}{2}}\right) .
\end{aligned}
$$

Proof. This is a straightforward computation using, e.g., [13] p. 249. In particular the presence of the Maslov index $\sigma_{\hat{\gamma}}$ comes from the square root already mentioned, as explained in [13] p. 239.

Remark. One can easily check that if we take a symbol $a_{m}^{R}=M(R) a_{m}$, where $a_{m}(\eta)=H_{m}(\eta) e^{-\eta^{2} / 2}$ with $H_{m}, m \in \mathbb{N}^{n-1}$, a Hermite polynomial, one gets in the elliptic case the following result:

$$
\begin{aligned}
\sum_{j} \varphi\left(\frac{E_{j}(\hbar)-E}{\hbar}\right)\left|\left(\psi_{(x, \xi)}^{a_{m}^{R}}, \psi_{j}^{\hbar}\right)\right|^{2} \\
=\frac{(4 \pi \hbar)^{-\frac{1}{2}}}{|\nabla \mathscr{H}(x, \xi)|} \sum_{k} \varphi\left(\left(k 2 \pi-\frac{S_{\gamma}}{\hbar}+\mathrm{Sub}_{\gamma}+\sigma_{\gamma}+\sum m_{j} \theta_{j}+\frac{1}{2} \sum_{j=1}^{n-1} \theta_{j}\right) / T_{\gamma}\right) \\
\quad+O\left(\hbar^{\frac{1}{2}}\right) .
\end{aligned}
$$

From this the computation for an arbitrary symbol $a$ follows, by expanding the symbol on the Hermite basis. 


\section{Discussion of the Results}

Our main theorems describe the weak asymptotics for small $\hbar$ of the weighted spectral measure

$$
\rho_{x, \xi}^{a}(\lambda):=\sum_{j} \delta\left(\frac{E_{j}(\hbar)-E}{\hbar}-\lambda\right)\left|\left(\psi_{(x, \xi)}^{a_{R}^{m}}, \psi_{j}^{\hbar}\right)\right|^{2}
$$

on the class $\mathscr{R}$ of Schwartz functions with compactly supported Fourier transform. Observe that the construction of $\rho$ is covariant as $(x, \xi)$ moves along the trajectory, $\gamma$, of the Hamilton flow of $\mathscr{H}$, in the sense that there is a symbol $a(t)$ such that

$$
\rho_{x(t), \xi(t)}^{a(t)}(\lambda)=\rho_{x, \xi}^{a}(\lambda),
$$

where $a_{0}(t)$ is the image of $a_{0}$ by the metaplectic quantization of the differential of the flow $d\left(\phi_{t}\right)_{(x, \xi)}$. Accordingly, the main properties of $\rho_{(x, \xi)}^{a}$ are related to the mechanical properties of $\gamma$. We wish here to explore this relationship, mainly through the Poisson-type formulae of the previous section.

In order to simplify the formulae a little we will suppose in this section that $\mathscr{H}_{s u b}=0$ (which is the ease for example for a classical Schrödinger operator, $H_{\hbar}=$ $\left.-\hbar^{2} \Delta+V\right)$. The formulae in the case $\mathscr{H}_{\text {sub }} \neq 0$ can be easily recovered. ${ }^{3}$

The fully elliptic case. We will see that in the fully elliptic case the weighted spectral measure is asymptotic to an analogous measure constructed from the quasimodes associated with $\gamma$. Recall (see $[8,4,18,15]$ ) that one can construct quasimodes associated to any elliptic closed trajectory, to first order. The construction can be summarized as follows. Let $\gamma$ be an elliptic trajectory of energy $E$ and let $(x, \xi) \in \gamma$. To $(x, \xi)$ and a symbol $a$ we associate the vector

$$
\Phi_{\gamma}^{a}=C \int_{0}^{T_{\gamma}} e^{i \frac{l(t)-E t}{\hbar}} \psi_{(x(t), \xi(t))}^{a(t)} d t
$$

where $a(t)=M(S(t))(a)$. By Theorem 3.1 we have that for some $a^{\prime}$

$$
i \hbar \partial_{t}\left(e^{i \frac{i(t)-E t}{\hbar}} \psi_{(x(t), \xi(t))}^{a(t)}\right)=\left(H_{\hbar}-E\right)\left(e^{i \frac{\left(\langle t)-E_{t}\right.}{\hbar}} \psi_{(x(t), \xi(t))}^{a(t)}\right)+\psi_{(x(t), \xi}^{\left.a^{\prime}(t)\right)} O\left(\hbar^{3 / 2}\right) .
$$

From this we get that

$$
\begin{aligned}
\left(H_{\hbar}-E\right) \Phi_{y}^{\alpha} & =i \hbar \int_{0}^{T_{y}} \partial_{t}\left(e^{i \frac{\eta(t)-E t}{\hbar}} \psi_{(x(t), \xi \xi(t))}^{\alpha(t)}\right)+O\left(\hbar^{3 / 2}\right) \\
& =i \hbar\left(e^{i \frac{S_{y}}{\hbar}} \psi_{(x, \xi)}^{a\left(T_{\gamma}\right)}-\psi_{(x, \xi)}^{a}\right)+O\left(\hbar^{3 / 2}\right) .
\end{aligned}
$$

Therefore $\left(H_{\hbar}-E\right) \Phi_{\gamma}^{a}=O\left(\hbar^{3 / 2}\right)$ provided that:

i) $M\left(S\left(T_{\gamma}\right)\right) a_{0}=e^{i \lambda} a_{0}$, and

ii) $\lambda=\frac{S_{y}}{\hbar}+2 \pi k$ for some integer $k$.

Here $a_{0}$ is the leading term of $a$ and $S_{y}$ the action of $y$. A solution to this problem is precisely given by $a=a_{R}^{m}, m \in \mathbb{Z}^{n}$, defined in the preceding section. Moreover such

\footnotetext{
${ }^{3}$ We will also assume for simplicity that $\mu=0$ in (116)
} 
an $a_{R}^{m}$ gives $\lambda=\sigma_{y}+\sum m_{j} \theta_{j}+\frac{1}{2} \sum \theta_{j}$, so (ii) is nothing but the Bohr-Sommerfeld condition (see [18]):

$$
S_{\gamma}=\left(2 \pi k+\sum m_{j} \theta_{j}+\sigma_{\gamma}+\frac{1}{2} \sum \theta_{j}\right) \hbar .
$$

The computation of the constant $C$ shows that if $\Phi_{\gamma}^{a}$ is normalized one has:

$$
\left|\left\langle\psi_{x, \xi}^{a_{R}^{m}}, \Phi_{\gamma}^{a_{R}^{m^{\prime}}}\right\rangle\right|^{2}=\frac{(4 \pi \hbar)^{-\frac{1}{2}}}{T_{\gamma}|\nabla \mathscr{H}(x, \xi)|} \delta_{m m^{\prime}}+O\left(\hbar^{\frac{1}{2}}\right) .
$$

Let us suppose now that $\gamma$ is non-degenerate on $\Sigma_{E}$, so it belongs to a family $\left\{\gamma_{s}\right\}$ of elliptic trajectories indexed by their action, $s$, where $s$ ranges in a neighborhood of $S_{\gamma}$. Let $E(s)$ and $T(s)$ denote the energy and the period of $\gamma_{s}$. Then the energies of the quasi-modes associated with this family are

$$
\mathscr{E}^{ø k, m}=E\left(\left(2 \pi k+\sum\left(m_{j}+\frac{1}{2}\right) \theta_{j}+\sigma_{\gamma}\right) \hbar\right) .
$$

Moreover it is well-known that

$$
\frac{d E(s)}{d s}=\frac{1}{T(s)} .
$$

Therefore, there is a smooth function $v(s)$ such that

$$
\mathscr{E}^{c k, m}-E=\frac{1}{T_{\gamma}}\left(\left(2 \pi k+c_{m}\right) \hbar-S_{\gamma}\right)+\left(\left(2 \pi k+c_{m}\right) \hbar-S_{\gamma}\right)^{2} v\left(\left(2 \pi k+c_{m}\right) \hbar-S_{\gamma}\right),
$$

where $c_{m}:=\sum\left(m_{j}+\frac{1}{2}\right) \theta_{j}+\sigma_{\gamma}$. Let us define the numbers $E_{Q M}^{k, m}$ by the equation

$$
\frac{E_{Q M}^{k, m}(\hbar)-E}{\hbar}=\frac{1}{T_{\gamma}}\left(2 \pi k+\sum m_{j} \theta_{j}+\sigma_{\gamma}+\frac{1}{2} \sum \theta_{j}-\frac{S_{\gamma}}{\hbar}\right) .
$$

Then (130) shows that, $\forall c>0$, as $\hbar \rightarrow 0$ and for $k$ 's such that $\left|2 \pi k \hbar-S_{\gamma}\right|<c \hbar$, one has

$$
\mathscr{E}^{k, m}=E_{Q M}^{k, m}+O\left(\hbar^{2}\right) \text {. }
$$

In other words, in a neighborhood of $\gamma$ one can find quasi-modes $\Psi_{k}^{m}$ and energies $E_{Q M}^{k, m}$ belonging to an interval of size $\hbar$ around $E$ satisfying

$$
H_{\hbar} \Psi_{k}^{m}=E_{Q M}^{k, m} \Psi_{k}^{m}+O\left(\hbar^{2}\right)
$$

for large $k\left(\left|2 \pi k \hbar-S_{\gamma}\right|<c \hbar\right)$. On the other hand, the right-hand side of (131) is precisely the argument of $\varphi$ in the right-hand side of (121). Taking into account (127) we can summarize as follows:

Proposition 7.1. Let $\gamma$ be elliptic and let $E_{Q M}^{k, m}(\hbar)$ defined by (131). Then, as functionals on the class $\mathscr{R}$ defined in Sect. 4 ,

$$
\rho_{x, \xi}^{a_{m}^{R}}(\lambda)=\sum_{k, m^{\prime}} \delta\left(\frac{E_{Q M}^{k, m^{\prime}}(\hbar)-E}{\hbar}-\lambda\right)\left|\left\langle\psi_{x, \xi}^{a_{R}^{m}}, \Phi_{\gamma}^{a_{R}^{m^{\prime}}}\right\rangle\right|^{2}+T,
$$


where $T$ is $O\left(\hbar^{\frac{1}{2}}\right)$ in the weak topology. Moreover, the $E_{Q M}^{k, m}(\hbar)$ are asymptotic to the energies of the quasi-modes $\Phi^{a_{R}^{m}}$ in the sense of (132).

Remark. One also has a similar result for the weighted spectral measure for an arbitrary symbol $a$, by decomposing $a$ on the Hermite basis, in which case the summation over $m^{\prime}$ would be non-trivial.

The unstable case. Let us turn now to the case where $\gamma$ is unstable.

Proposition 7.2. If $\gamma$ is fully hyperbolic then on the class $\mathscr{R}$ the weighted spectral measure $\rho_{x, \xi}^{a^{R}}$ (where $a^{R}$ is the Gaussian (117)) is, modulo $\hbar^{\frac{1}{2}}$ (also in the weak topology), Lebesgue-continuous of the form $g(\lambda) d \lambda$, where

$$
g(\lambda)=\frac{(4 \pi)^{-\frac{1}{2}}}{T_{\gamma}|\nabla \mathscr{H}(x, \xi)|} \sum_{-\infty}^{+\infty} h\left(\frac{1}{T_{\gamma}}\left(2 \pi k-\frac{S_{\gamma}}{\hbar}+\sigma_{\gamma}\right)-\lambda\right)
$$

with

$$
h(\lambda)=\frac{1}{\sqrt{2 \pi}} \int \frac{e^{i \lambda t}}{\sqrt{\prod_{0}^{n-1} \cosh \left(\mu_{k} t\right)}} d t .
$$

Proof. By the results of Sect. 6 in this case the coefficient $d_{0}$ has the form:

$$
\frac{(4 \pi)^{-\frac{1}{2}}}{|\nabla \mathscr{H}(x, \xi)|}\left(\sum_{l} \frac{1}{\sqrt{\prod_{k=1}^{n-1} \cosh \left(l \mu_{k}\right)}} \hat{\varphi}\left(l T_{y}\right)\right) .
$$

By the Poisson summation formula, and using the fact that the Fourier transform of a product is a convolution, one gets the result.

Remark. The formula above is, for small Liapunov exponents, a kind of "smearedout Poisson formula." As shown by (136), $h(\lambda) \rightarrow \delta(\lambda)$ as all the Liapunov exponents $\mu_{k}$ tend to 0 . This shows that $g(\lambda)$ has peaks around the lattice $\frac{1}{T_{y}}(2 \pi k-$ $\frac{S_{y}}{\hbar}+\sigma_{\gamma}$ ) if $\gamma$ is not too unstable.

We would like to finish with two informal remarks:

1. No construction of quasi-modes is available in the unstable case; nevertheless numerical computations (see $[12,6,9]$ ) show that some phenomenon of localization of eigenfunctions near unstable periodic orbits are visible. Among the main properties of this controversial "scarring" phenomenon we point out:

- The apparent localization doesn't occur more strongly as $\hbar$ goes to 0 .

- This localization is more visible if the Liapunov exponents of $\gamma$ are small.

Our results show that the average over a band of energy of the Husimi functions (namely $\left|\left(\psi_{(x, \xi)}^{a}, \psi_{j}^{\hbar}\right)\right|^{2}$ ) share some of those properties. Corollary 1.4 shows that there is a nonzero density of eigenfunctions whose Husimi functions are actually pointwise sensitive to the presence of periodic trajectories. The contributions to the formulas of Theorems 1.2 and 1.4 are in accordance with the properties of scars mentioned before:

- The fact that $(x, \xi)$ belongs or not to a periodic trajectory doesn't affect the order of the expansion, but rather changes the numerical leading coefficient.

- This coefficient becomes greater as $\mu$ (the highest Liapunov exponent) tends to 0 . 
2. We finish by discussing the dependence of the leading coefficient $d_{0}$ with respect to the symbol $a$. The extra contribution of a hyperbolic $\gamma$ to the leading term of $\sum_{j} \varphi\left(\frac{E_{j}(\hbar)-E}{\hbar}\right)\left|\left(\psi_{(x, \xi)}^{a}, \psi_{j}^{\hbar}\right)\right|^{2}$ is

$$
b(a):=\sum_{l \neq 0} \hat{\varphi}\left(l T_{\gamma}\right) e^{i \frac{l S_{y}}{\hbar}} \int e^{-i i^{2} \dot{x} \dot{\xi}} e^{i t h x} \overline{a(\eta)}\left(U^{l} a\right)(\eta-t \dot{\xi}) d \eta d t .
$$

Let us take $(x, \xi) \in \gamma$ and let us suppose $n=2$ (the case $n>2$ can be treated analogously). Let $a_{0}=(4 \pi)^{-1 / 2} e^{-\eta^{2} / 2}$. We wish to estimate $\left(a_{0}, U^{l} Z(t \dot{x}, t \dot{\xi}) a_{0}\right)$. First remark that

$$
\left|\left(a, a^{\prime}\right)\right|^{2}=\int \mathscr{W}_{a}(u, v) \mathscr{W}_{a^{\prime}}(u, v) d u d v,
$$

where $\mathscr{W}_{a}$ is the Wigner function of $a$. Moreover it is well known that

$$
\mathscr{W}_{M(S) Z(e, f) a}(u, v)=\mathscr{W}_{a}\left(S^{-1}(u-e, v-f)\right) .
$$

$\mathscr{W}_{a_{0}}$ has an effective support of size 1 near the origin, since

$$
\mathscr{W}_{a_{0}}=e^{-\left(u^{2}+v^{2}\right)} .
$$

Consider now $(e, f)=b_{s}+b_{u}$ with $b_{s}$ and $b_{u}$ tangent vectors belonging to the stable and unstable directions at $(x, \xi)$. Then $\mathscr{W}_{Z(e, f)} a_{0}$ will have an effective support near $b_{s}+b_{u}$. It is easy to see that the effective support of $\mathscr{W}_{U^{l} Z(e, f) a_{0}}$ won't intersect the one of $\mathscr{W}_{Z(e, f) a_{0}}$ as soon as:

$$
\left|b_{s}\right|>\frac{1+e^{\mu}}{1-e^{\mu}}
$$

or

$$
\left|b_{u}\right|>\frac{1+e^{-\mu}}{1-e^{-\mu}} .
$$

If one remarks finally that

$$
\psi_{(x, \xi)}^{Z(e, f) a}(y)=\psi_{(x, \xi)}^{a}(y-\sqrt{\hbar} e) e^{-i \frac{\sqrt{\hbar} f \underline{\zeta}}{\hbar}},
$$

i.e. $\psi_{(x, \xi)}^{Z(e, f) a}$ is microlocalized around the point $(x+\sqrt{\hbar} e ; \xi+\sqrt{\hbar} f)$, one may conclude as follows:

If the "effective support" of a is roughly of size 1 and contains the origin, then $\sum_{j} \varphi\left(\frac{E_{j}(\hbar)-E}{\hbar}\right)\left|\left(\psi_{(x, \xi)}^{a}, \psi_{j}^{\hbar}\right)\right|^{2}$ is sensitive to the presence of $\gamma$ in a tubular neighborhood of $\gamma$ of size $\sqrt{\hbar} \frac{e^{\mu}+1}{e^{\mu}-1}$ in the stable direction and $\sqrt{\hbar} \frac{e^{-\mu}+1}{1-e^{-\mu}}$ in the unstable one.

\section{References}

1. Boutet de Monvel, L., Guillemin, V.: The spectral theory of Toeplitz operators. Annals of Mathematics Studies No. 99, Princeton, New Jersey: Princeton University Press, 1981

2. Brummelhuis, R., Paul, T., Uribe, A.: Spectral estimates near a critical level. To appear in Duke J. Math.

3. Brummelhuis, R., Uribe, A.: A trace formula for Schrödinger operators. Commun. Math. Phys. 136, 567-584 (1991) 
4. Colin de Verdière, Y.: Quasi-modes sur les varietes Riemanniennes. Invent. Math. 43, 15-42 (1977)

5. Cordoba, A., Fefferman, C.: Wave packets and Fourier integral operators. Commun. in P.D.E. 23, 979-1005 (1978)

6. Delande, D.: Commun. Atom. Mol. Phys. 25 (1991)

7. Grossmann, A.: Parity operator and quantization of $\delta$ functions. Commun. Math. Phys. 48, 191-194 (1976)

8. Guillemin, V.: Symplectic spinors and partial differential equations. In: Coll. Inst. CNRS n. 237, Géométrie Symplectique et Physique Mathématique, pp. 217-252

9. Gutzwiller, M.: Chaos in classical and quantum Mechanics. Berlin-Heidelberg-New York: Springer-Verlag, 1991

10. Hagedorn, G.: Semiclassical quantum mechanics. Commun. Math. Phys. 71, 77-93 (1980)

11. Helffer, B., Martinez, A., Robert, D.: Ergodicité et limite semi-classique. Commun. Math. Phys. 109, 313-326 (1987)

12. Heller, E.: Phys. Rev. Lett. 53, 1515 (1984)

13. Litteljohn, R.: The semiclassical evolution of wave packets. Phys. Repts. 138, 193-291 (1986)

14. Paul, T., Uribe, A.: The semi-classical trace formula and propagation of wave packets. (To appear in J. Funct. Anal.)

15. Ralston, J.V.: On the construction of quasimodes associated with stable periodic orbits. Commun. Math. Phys. 51, 219-242 (1976)

16. Taylor, M.: Pseudodifferential operators. Priceton, NJ: Princeton University Press, 1981

17. Treves, F.: Introduction to pseudodifferential and Fourier integral operators. New York and London: Plenum Press, 1980

18. Voros, A.: The WKB-Maslov method for nonseparable systems. In: Coll. Inst. CNRS n. 237, Géométrie Symplectique et Physique Mathématique, pp. 217-252

Communicated by G. Felder 